APJ DRAFT, 22 JUNE 2017

Preprint typeset using LTEX style emulateapj v. 05/12/14

\title{
TYCHO-GAIA ASTROMETRIC SOLUTION PARALLAXES AND PROPER MOTIONS FOR FIVE GALACTIC GLOBULAR CLUSTERS
}

\author{
LAURA L. WATKINS AND ROELAND P. VAN DER MAREL \\ Space Telescope Science Institute, 3700 San Martin Drive, Baltimore MD 21218, USA \\ ApJ draft, 22 June 2017
}

\begin{abstract}
We present a pilot study of Galactic globular cluster (GC) proper motion (PM) determinations using Gaia data. We search for GC stars in the Tycho-Gaia Astrometric Solution (TGAS) catalogue from Gaia Data Release 1 (DR1), and identify five members of NGC 104 (47 Tucanae), one member of NGC 5272 (M 3), five members of NGC 6121 (M 4), seven members of NGC 6397, and two members of NGC 6656 (M 22). By taking a weighted average of member stars, fully accounting for the correlations between parameters, we estimate the parallax (and, hence, distance) and PM of the GCs. This provides a homogeneous PM study of multiple GCs based on an astrometric catalogue with small and well-controlled systematic errors and yields random PM errors similar to existing measurements. Detailed comparison to the available Hubble Space Telescope (HST) measurements generally shows excellent agreement, validating the astrometric quality of both TGAS and HST. By contrast, comparison to ground-based measurements shows that some of those must have systematic errors exceeding the random errors. Our parallax estimates have uncertainties an order of magnitude larger than previous studies, but nevertheless imply distances consistent with previous estimates. By combining our PM measurements with literature positions, distances, and radial velocities, we measure Galactocentric space motions for the clusters and find that these also agree well with previous analyses. Our analysis provides a framework for determining more accurate distances and PMs of Galactic GCs using future Gaia data releases. This will provide crucial constraints on the near end of the cosmic distance ladder and provide accurate GC orbital histories.

Keywords: globular clusters: general - globular clusters: individual (NGC 104 (47 Tucanae), NGC 5272 (M 3), NGC 6121 (M 4), NGC 6397, NGC 6656 (M 22)) - parallaxes - proper motions - stars: kinematics and dynamics
\end{abstract}

\section{INTRODUCTION}

Accurate distances and space motions of Galactic globular clusters (GCs) are difficult but incredibly valuable to measure: improved constraints will have implications for the origin and evolution of the clusters themselves, for the structure of the Milky Way (MW), and for the fine-tuning of cosmological models.

The prevailing theory for the origin of the MW's GC population is that some GCs were formed outside of the MW and later accreted while the rest were formed in situ (e.g., Mackey $\&$ Gilmore 2004). The different origins and histories for the accreted and in-situ populations will manifest in their orbits (e.g., Zhu et al. 2016) and imprint on their internal dynamics (e.g., Webb et al. 2014; Zocchi et al. 2016), so determining which GCs belong to which population and analysing the differences between them will advance our understanding of cluster formation and evolution.

Moreover, accurate six-dimensional phase-space information for the GCs will allow us to constrain cluster orbits (e.g., Küpper et al. 2015), which will indicate which clusters have been most affected by tides and if any known GCs could be the missing progenitors of tidal streams whose origin is unknown. Cluster orbits will also be beneficial in constraining the inner shape of the Galactic halo (e.g., Pearson et al. 2015).

Local GCs populate the short end of the cosmological distance ladder, so improved distances will provide important cosmology-independent verification for cosmological models (Verde et al. 2013). Distances are also a key ingredient in GC age determination using the luminosity of the main-sequence (MS) turn off (e.g. Chaboyer 1995) and currently, the distance

lwatkins@stsci.edu uncertainties in such analyses dominate over other sources of error. Accurate GC ages are of special interest as GCs are among the oldest objects in the universe for which ages are known and so help to constrain the age of the universe (e.g., Krauss \& Chaboyer 2003).

Not only are distances and proper motions (PMs) useful measurements to have, but multiple measurements, using different data types and analysis methods, improve accuracy and ensure that the measurements are not biased. GC distances are typically estimated photometrically using "standard candles", such as RR Lyraes or the position of the horizontal branch (HB). However, it is also possible to estimate distances from kinematic data by comparing line-of-sight velocity and PMs measurements. In Watkins et al. (2015a), we estimated dynamical distances for 15 Galactic GCs using the Hubble Space Telescope (HST) Proper Motion (HSTPROMO) ${ }^{1}$ GC catalogues (Bellini et al. 2014) and compared them to the photometric distance estimates compiled in Harris (1996, 2010 edition, hereafter H96). Overall we showed that the two very different methods of distance estimation agreed very well, implying that both methods are robust and unbiased, however, a further comparison against parallax distances will provide a useful additional test.

Absolute PMs have previously been measured from the ground for 63 Galactic GCs (Dinescu et al. 1997, 1999b,a, 2003; Casetti-Dinescu et al. 2007, 2010, 2013, hereafter CD13) $)^{2}$ as part of the Southern Proper Motion (SPM) Program. These measurements are heterogeneous as the method used to correct the PMs to an inertial reference frame varied

\footnotetext{
${ }^{1}$ http://www.stsci.edu/ marel/hstpromo.html

${ }^{2}$ A compilation is available at http://www.astro.yale.edu/dana/gc.html.
} 
from cluster to cluster. Also, in some cases, the measurements represent a combination of multiple determinations (by including other PM determinations from other ground- and space-based sources) and not a single determination. Nevertheless, independent PM measurements for these GCs will be incredibly helpful to verify their accuracy and to assess the consistency of the different methods.

Gaia will provide five-parameter astrometric solutions positions, parallaxes, and PMs - for objects brighter than $\sim 20 \mathrm{mag}$, so it will be tremendously useful for analysing both internal and global PMs for Galactic GCs and their distances $^{3}$. PM and parallax are both degenerate, and a sufficiently long baseline is needed to disentangle the two; at the time of the first Gaia data release (DR1, 2016 September, Gaia Collaboration et al. 2016), parallaxes and PMs were not measurable with Gaia data alone. The first Gaia-only PM measurements are projected for release in late 2017 and the final (and most accurate) PMs will be released only in 2022. However, by crossmatching the Gaia catalogue with the Hipparcos Tycho2 catalogue (Høg et al. 2000), it is possible to measure PMs (and parallaxes) for stars in both datasets: the Tycho-Gaia Astrometric Solution (TGAS, Michalik et al. 2015; Lindegren et al. 2016). Combining Tycho2 and Gaia data together offers an extended baseline for PM studies that has made PM analyses achievable for a few million stars with the Gaia DR1. Although not ideally suited to dynamical studies of objects within the Local Group, TGAS has already been used to measure the rotation fields of the Magellanic Clouds (van der Marel \& Sahlmann 2016) and to measure the space motion of GC NGC 2419 (Massari et al. 2017). Here, we search for Galactic GC stars in the TGAS catalogue.

This is a pilot study based on the limited information currently available. We hope that our methods can serve as a template for studies with future Gaia data releases, which will have more stars and will yield higher accuracy PM results.

We outline our cluster-member determination in Section 2, present our parallax and PM results in Section 3, discuss the implied space motions and orbits of the clusters in Section 4, and conclude in Section 5.

\section{CLUSTER MEMBERSHIP DETERMINATION}

To determine likely GC members, we search for stars that are close to a GC centre on the plane of sky and then check for consistency with previous PM and parallax estimates and typical GC isochrones. We also wish to consider predictions for the PMs, parallaxes, and photometry expected for MW stars along the line of sight of the GC to rule out contaminants.

\subsection{Proximity}

To estimate how many Galactic GC stars may exist in the TGAS catalogue, we count the number of stars found within some limiting radius from the centre of each $\mathrm{GC}$ on the plane of the sky. We begin with the H96 Galactic GC catalogue from which we extract cluster centre coordinates, concentrations, and core radii. Although the catalogue contains data for 157 Galactic GCs, we were only able to proceed with 156 GCs, since Pyxis has no concentration or core radius estimate listed. $^{4}$

\footnotetext{
${ }^{3}$ Note that Astraatmadja \& Bailer-Jones (2016) caution that estimation of distances from Gaia parallaxes is not as simple as inverting the parallaxes, since the parallax uncertainties are non-negligible and must be done using rigorous statistical analysis.

${ }^{4}$ This does not affect our analysis, as Pyxis is too distant to have stars with sufficient apparent brightness in the Tycho2 catalogue.
}

We use the concentrations $c$ and core radii $R_{\text {core }}$ to estimate the tidal radii $R_{\text {tidal }}$ of the GCs via

$$
R_{\text {tidal }}=10^{c} R_{\text {core }} .
$$

This is not generally considered to be a robust method of tidalradius determination and is likely to underestimate the true extent of a GC. These tidal radii estimates assume an underlying King profile, however, GCs are generally better fit by Wilson models (e.g., McLaughlin \& van der Marel 2005), which are still finite in extent but are more extended than King models. Furthermore, a GC that is tidally disrupting may have member stars outside of its formal tidal radius. However, this method is sufficient for our purposes, which is simply to estimate the approximate extent of the clusters on the plane of the sky. To mitigate the possibility of underestimating the true extent of the cluster, we adopt $2 R_{\text {tidal }}$ as the limiting radius within which to search for GC members.

Then, for each GC in turn, we extracted all stars within $2 R_{\text {tidal }}$ of the cluster centre from the TGAS catalogue. We did this for all 156 GCs with no cuts on heliocentric distance, magnitude, or central velocity dispersion (all of which could affect the likelihood that a star close to the centre in projection is a true cluster member). In total, we identified 4268 stars within $2 R_{\text {tidal }}$ of the centre of a GC across 142 clusters.

\subsection{Distance and Extinction}

Some of these clusters are relatively far away and others are in regions of high extinction, so it is likely that true GC members will be rendered too faint for detection with TGAS. So we wish to determine the expected apparent magnitude of the brightest star in any given GC and then the number of observed stars fainter than this limit.

To do this, we select the $V_{\mathrm{T}}$ magnitude from the Tycho 2 catalogue or the $H_{p}$ magnitude from the Hipparcos catalogue for each star, depending on the original catalogue of origin. Although there is not a perfect correspondence, both Hipparcos $H_{p}$ and Tycho2 $V_{\mathrm{T}}$ can be approximated by the $H S T$ F555W filter, for which we have isochrones and extinction coefficients. We then take a typical GC isochrone from the Dartmouth Stellar Evolution Database (Dotter et al. 2008) with metallicity $[\mathrm{Fe} / \mathrm{H}]=-1.5 \mathrm{dex}$, alpha-element abundance $[\alpha / \mathrm{Fe}]=0.2 \mathrm{dex}$, and age $A=11 \mathrm{Gyr}$, and estimate the brightest magnitude that the isochrone reaches.

For each cluster, we adjust the tip magnitude of the isochrone for GC distance and extinction along the line of sight using distance moduli calculated using distances from H96, extinctions also from H96, and extinction coefficients from Sirianni et al. (2005). To allow for magnitude uncertainties, we set the limiting magnitude at 0.5 mag brighter than the distance- and extinction-corrected tip of the isochrone and count the number of stars fainter than this limiting magnitude. We perform a more careful photometry check on our GC member candidates in Section 2.5, this rather crude preliminary cut is intended to remove distant and highly extincted clusters from further consideration. After these cuts, we are left with 967 stars across 30 clusters; the most distant of these clusters lies at $10.3 \mathrm{kpc}$.

\subsection{Parallax and Proper Motion}

To determine which of these stars are likely to be cluster members, we wish to identify which stars have parallax and PM measurements that are consistent with previous measurements for their nearby cluster. 
TGAS provides parallaxes $\varpi$ and PMs $\left(\mu_{\alpha *}, \mu_{\delta}\right)$ for each star, along with uncertainties for each and correlation coefficients among the parameters, from which we can construct a full 3-dimensional covariance matrix.

For the GCs, we adopt distances from H96, which we then invert to determine parallax estimates. The H96 distances have no formal error bars, so we adopt errors of $10 \%$ on our parallax estimates. For PM measurements, we use values from the compilation described in CD13 and earlier papers, which themselves come from a variety of sources and are sometimes averages of multiple measurements. Only 26 of the 30 clusters with GC member candidates have PM measurements in this compilation and so only these 26 GCs can be evaluated here.

For each cluster, we calculate the probability for the nearby stars of being a cluster member. For a star $i$ with parallax and PM measurements $\mathbf{m}_{i}$ and covariance $\mathbf{C}_{i}$, we ask what the likelihood $\mathcal{L}_{\varpi \mu, \mathrm{i}}$ is that this star is a member of a GC with measurements $\mathbf{m}_{\mathrm{GC}}$ and covariance $\mathbf{C}_{\mathrm{GC}}$,

$$
\begin{aligned}
\mathcal{L}_{\varpi \mu, \mathrm{i}}= & p\left(\mathbf{m}_{\mathrm{i}} \mid \mathbf{C}_{\mathrm{i}}, \mathbf{m}_{\mathrm{GC}}, \mathbf{C}_{\mathrm{GC}}\right) \\
= & \frac{\exp \left[-\frac{1}{2}\left(\mathbf{m}_{\mathrm{i}}-\mathbf{m}_{\mathrm{GC}}\right)^{\mathrm{T}}\left(\mathbf{C}_{\mathrm{i}}+\mathbf{C}_{\mathrm{GC}}\right)^{-1}\left(\mathbf{m}_{\mathrm{i}}-\mathbf{m}\right.\right.}{\sqrt{(2 \pi)^{3}\left|\left(\mathbf{C}_{\mathrm{i}}+\mathbf{C}_{\mathrm{GC}}\right)\right|}}
\end{aligned}
$$

which is a standard 3-dimensional Gaussian. To construct the GC covariance matrix $\mathbf{C}_{\mathrm{GC}}$, we assume that the errors are uncorrelated, so the diagonal terms are the squared uncertainties on the parallax and PM measurements and the off-diagonal elements are zero. We further add the GC velocity dispersion (H96) in quadrature to the PM terms to account for the expected spread in velocities.

This likelihood calculation assumes that all stars selected near the cluster are equally likely to be members, but this is not the case. Stars close to the cluster centre of the cluster are more likely to be members than stars near to the $2 R_{\text {tidal }}$ boundary, so we also calculate the likelihood of a star $i$ with coordinates $\left(\alpha_{\mathrm{i}}, \delta_{\mathrm{i}}\right)$ being a member of the GC with centre $\left(\alpha_{\mathrm{GC}}, \delta_{\mathrm{GC}}\right)$ as

$$
\begin{aligned}
\mathcal{L}_{\alpha \delta, \mathrm{i}} & =p\left(\alpha_{\mathrm{i}}, \delta_{\mathrm{i}} \mid \alpha_{\mathrm{GC}}, \delta_{\mathrm{GC}}, \sigma\right) \\
& =\exp \left[-\frac{1}{2 \sigma^{2}}\left(\left(\alpha_{\mathrm{i}}-\alpha_{\mathrm{GC}}\right)^{2}+\left(\delta_{\mathrm{i}}-\delta_{\mathrm{GC}}\right)^{2}\right)\right] .
\end{aligned}
$$

where we use $\sigma=\frac{1}{2} R_{\text {tidal }}$ to account for the approximate extent of the cluster. The uncertainties of the cluster centre coordinates and of the positions of the stars are negligible compared to the extent of the cluster, so we neglect the measurement errors in this calculation.

GC projected number density profiles are, of course, highly non-Gaussian, however, the profiles in the outer regions of GCs are largely uncertain due to effect of tides and the scarcity of data there. This Gaussian weighting suffices to down-weight stars found further away from the cluster centre. Also note that we have used unnormalised Gaussians here, as we are mostly concerned with how many tidal radii the star is away from the cluster centre, rather than the tidal radius itself. That is, a star $1 R_{\text {tidal }}$ from the cluster centre should have the same $\mathcal{L}_{\alpha \delta, \mathrm{i}}$ in all clusters, regardless of the value of $R_{\text {tidal }}$.

Finally, we can calculate the total likelihood that the star is a member of the cluster $\mathcal{L}_{\mathrm{i}}=\mathcal{L}_{\varpi \mu, \mathrm{i}} \times \mathcal{L}_{\alpha \delta, \mathrm{i}}$. We keep all stars

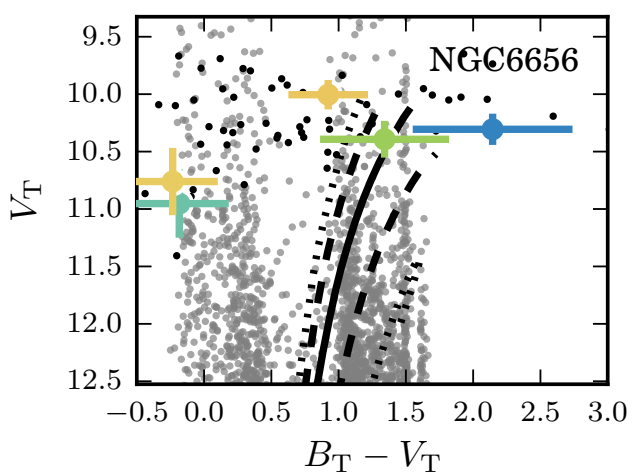

Figure 1. Representative distance-corrected isochrones for NGC 6656. The solid line shows an $11 \mathrm{Gyr},[\alpha / \mathrm{Fe}]=0.2$ dex isochrone with $[\mathrm{Fe} / \mathrm{H}]$ metallicity from $\mathrm{H} 96$ rounded to the nearest $0.5 \mathrm{dex}$, the dashed lines show the isochrones at $\pm 0.5 \mathrm{dex}$, and the dotted lines show the isochrones at $\pm 1 \mathrm{dex}$. The extinction-corrected TGAS cluster-member candidates are shown as coloured points where the colour indicates their likelihood from high (orange) to low (blue). The black points are TGAS stars ruled out as cluster members. For comparison, we show the expected MW field-star population from Besançon model predictions as grey points. For this cluster, we reject the two stars with $B_{\mathrm{T}}-V_{\mathrm{T}}<0$, but consider the others to be candidate cluster members.

with $\mathcal{L}_{\mathrm{i}}>-11$ as possible cluster members. ${ }^{5}$ This selects 64 stars across 15 clusters. All of the identified candidates are Tycho2 stars, no Hipparcos stars remain in our sample.

\subsection{Radial Velocities}

As a further test for membership, we check whether the candidate cluster members have radial velocities (RVs) consistent with cluster membership. We cross-match our stars with the RAdial Velocity Experiment (RAVE, Kunder et al. 2017) catalogue to extract RVs and find 15 stars in common between our high-likelihood sample and RAVE. Using RV and velocity dispersion estimates from H96, we sigma-clip the sample at $3 \sigma$ and reject five stars.

This leaves 59 candidate cluster members across 15 clusters: 11 stars in NGC 6838 (M71); 8 stars in each of NGC 104 (47 Tuc) and NGC 6752; 7 stars in NGC 6397; 5 stars in each of NGC 5139 ( $\omega$ Centauri), NGC 6121 (M 4) and NGC 6656 (M 22); 2 stars in each of NGC 6218 (M 12) and NGC 6254; and 1 star only in each of NGC 4833, NGC 5272 (M 3), NGC 6362, NGC 6723 (M 19), NGC 6779 (M 56), and NGC 6809.

\subsection{Photometry}

Many of the identified clusters are classified as disk or bulge clusters, so it is likely that some stars identified as possible members are disk or bulge stars along the line of sight to the cluster. Furthermore, it is likely that high reddening would have made cluster stars too faint for detection with Tycho2. So the next step is to assess whether the stars are photometrically consistent with their nearest cluster. To do this, we wish to investigate whether the identified stars are consistent with an old isochrone at the known distance, reddening, and metallicity.

Once again, we cross-match with the Tycho 2 catalogue to extract $B_{\mathrm{T}}$ and $V_{\mathrm{T}}$ magnitudes, which are very close to the Johnson $B$ and $V$ magnitudes. We then extract representative

\footnotetext{
${ }^{5}$ We experimented with different likelihood cuts and found that the analysis is fairly insensitive to the precise likelihood cut value but that a cut at $\mathcal{L}_{\mathrm{i}}>-11$ works well.
} 
isochrones for each cluster from the Dartmouth Stellar Evolution Database (Dotter et al. 2008). To do this, we assume an $\alpha$-element abundance $[\alpha / \mathrm{Fe}]$ of $0.2 \mathrm{dex}$ and an age of $11 \mathrm{Gyr}$, and use $[\mathrm{Fe} / \mathrm{H}]$ metallicities from $\mathrm{H} 96$ rounded to the nearest 0.5 dex. We adjust the isochrone magnitudes for distance (using distance moduli calculated from distances in H96), and correct the observed magnitudes for extinction using reddening values from $\mathrm{H} 96$ and extinction coefficients from Sirianni et al. (2005), using the F435W filter to approximate $B_{\mathrm{T}}$ and the F555W filter to approximate $V_{\mathrm{T}}{ }^{6}$

As an example, in Figure 1, we show distance-corrected isochrones for NGC 6656. Results for the other 14 clusters are given in Appendix A. The solid line shows the isochrone with cluster $[\mathrm{Fe} / \mathrm{H}]$, the dashed lines show the isochrones at \pm 0.5 dex, and the dotted lines show the isochrones at \pm 1 dex. The TGAS stars identified as possible members are shown as coloured points, with their colours representing their likelihood of membership based on their position, parallax, and PM, from orange (high) to blue (low). TGAS stars already ruled out as members (and so likely MW foreground stars) are shown in black.

From a visual inspection of the resulting isochrones for each cluster, we find that the candidate cluster members in NGC 4833, NGC 6362, NGC 6723, and NGC 6779 are all significantly offset from the isochrones and so we remove these from further consideration. We also reject 1 star in NGC 6254, 2 stars in NGC 6656, 1 star in NGC 6752, and 3 stars in NGC 6838 that are all significantly bluer than the expected cluster isochrones. The other stars remain promising candidates, and we still have 48 possible members across 11 clusters.

\subsection{Besançon Simulations}

As a final test, we wish to compare the velocities of the TGAS stars against predictions from the Besançon simulations (Robin et al. 2003); these simulations serve as a prediction for what the population of field stars should look like - both photometrically and kinematically - along the line of sight to any given cluster.

We selected stars from the Besançon simulations using the webtool provided, and we found that all of the default inputs were suitable for our purposes. We used the small-field option to select stars and used Galactic longitudes and latitudes from H96 to identify the cluster centres.

As an example, in Figure 2, we show 2D projections of the parallax, RA PM and Dec PM space for NGC 6656. Results for the other 10 clusters are given in Appendix A. The red stars indicate the H96 distance inverted to calculate a parallax and the PMs from CD13. The coloured points are the candidate cluster members from TGAS, coloured according to their probability of membership from orange (high) to blue (low). The black points are TGAS stars selected inside $2 R_{\text {tidal }}$ rejected as cluster-member candidates (and so likely MW field stars). The grey points show the predictions for the MW field population from Besançon simulations. Note that the error bars are not aligned with the axes, as we have incorporated the correlation terms in the five-parameter astrometric solution provided in the TGAS catalogue. The error bars show the major and minor axes of the $2 \mathrm{D}$ projection of the full covariance matrix. In the top-right corners, we show sky position maps for the TGAS candidate cluster members (coloured

\footnotetext{
${ }^{6}$ These approximations are sufficient for present analysis, which does not rely on accurate photometry.
}

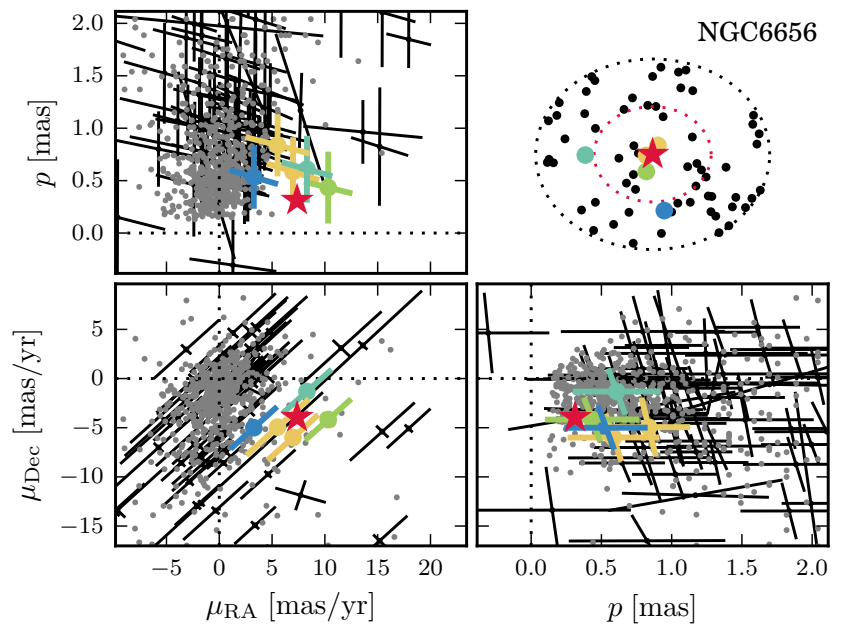

Figure 2. Sky positions, parallaxes, and proper motions for the TGAS stars in NGC 6656,. The top-right panel shows the sky positions of the TGAS stars identified within $2 R_{\text {tidal }}$ relative to the cluster centre (red star). The red dotted line marks $R_{\text {tidal }}$ and the black dotted line marks $2 R_{\text {tidal }}$. The remaining panels show projections of the five-parameter astrometric solution for parallax, RA PM and Dec PM. The literature values we used to determine membership probabilities (parallax from the inverted distance from $\mathrm{H} 96$ and PMs from CD13) are shown as red stars. The TGAS cluster-member candidates are shown as coloured points, coloured by their probability of membership from orange (high) to blue (low). The black points are TGAS stars rejected as cluster members, and thus, thought to be MW field stars. For comparison, Besançon model predictions are shown as grey points. Some of the Besançon stars fall outside of the plot limits.

points) and the TGAS field stars (black points) relative to the centre of the cluster (red star). The red dotted lines marks the tidal radii $R_{\text {tidal }}$, and the black dotted lines mark $2 R_{\text {tidal }}$.

We find that both the photometric and kinematic properties for 6 of the remaining 11 clusters are consistent with the Besançon predictions for the field-star population, and the other TGAS stars in the respective fields, which are also assumed to be field stars. In all cases, we cannot be confident that we have truly selected cluster members and not field stars, and so we remove these clusters from further consideration. The removed clusters are: NGC 5139, NGC 6218, NGC 6254, NGC 6752, NGC 6809, and NGC 6838.

Thus, we are left with five promising clusters, which we now discuss in turn.

NGC 104 has eight candidate stars, but it also poses the challenge that the Besançon model predictions overlap the part of parallax and PM space where NGC 104 stars are expected. Therefore, for this cluster we decided to restrict our final analysis to only the stars within $0.5 R_{\text {tidal }}$ from the centre, which is where the cluster-to-foreground star ratio should be largest. There are five stars that meet this criterion, and all of them clump in PM space around the expected NGC 104 PM value. While we cannot strictly rule out that any of these five stars are foreground, we can rule out that a significant fraction of them are foreground, since the Besançon models predict a much larger PM dispersion for such stars. One of the five candidate member stars also has an RV estimate within $3 \sigma$ of the cluster value (in fact, at $1.2 \sigma$ ). This does lend further credence to their membership (although we find that $65 \%$ of the Besançon stars also have RVs predicted to be within $3 \sigma$ of the cluster value).

NGC 5272 has only a single candidate, however, it is very close to the cluster centre and the agreement in PM and parallax is excellent. Furthermore, both cluster and star sit far 
away from the Besançon predictions. We believe that this star is a member of NGC 5272, though we acknowledge that it is hard to be certain for a single star.

NGC 6121 has five candidates; all lie well within the tidal radius and all have PMs and parallaxes consistent both with the cluster and each other and are inconsistent with the Besançon predictions. We note that one star is slightly offset from the others, however, its proximity to the centre and its position relative to a cluster isochrone both mark it as a promising candidate. Unfortunately, this offset star is not augmented by RV data, however, two other candidates do have RV estimates: one lies at $\sim 0.2 \sigma$ from the cluster value and the other at $\sim 1.1 \sigma$ from the cluster value, thus indicating that they are indeed bona fide cluster members. We believe all five stars are cluster members.

NGC 6397 has seven candidates, all of which are clearly offset from the bulk of the Besançon predictions in PM. One star (light blue) also has an RV measurement within $1 \sigma$ of the cluster measurement, however, $\sim 22 \%$ of the Besançon stars have RVs within $3 \sigma$ of the cluster value, so RV is not a strong indicator of cluster membership. Nevertheless, we believe that all seven stars are members.

NGC 6656 has three candidates, all of which have parallaxes and $\mu_{\delta}$ measurements consistent with the Besançon population; two (yellow and green) are clearly offset in $\mu_{\alpha *}$ from the bulk of the background predictions but the third (blue) does sit at the edge of the predicted background distribution; as this star also sits outside of the tidal radius and is somewhat redward of the expected cluster isochrones, we remove it from our analysis. Of the remaining two stars, the star coloured yellow has an RV measurement that is $2 \sigma$ offset from the cluster value. Fewer than $3 \%$ of the Besançon stars have RVs within $3 \sigma$ of the cluster velocity, so RV is indeed a strong indicator of cluster membership for this cluster. We believe that the remaining two stars are members.

In summary, we are confident that we have identified $20 \mathrm{GC}$ member stars: 5 stars in NGC 104, 1 star in NGC 5272, 5 stars in NGC 6121, 7 stars in NGC 6397, and 2 stars in NGC 6656. In Table 1, we provide the TGAS and Tycho 2 properties for all stars in these clusters that passed the parallax-PM likelihood cut, along with their known RVs, and the likelihoods calculated in Section 2.3. Stars marked with a $\star$ are those in the final sample that we believe to be bona fide members.

Perhaps unsurprisingly, NGC 6121, NGC 6397, and NGC 6656 are three of the four closest clusters. ${ }^{7}$ Thanks in part to their proximity, these clusters are among the best studied and so precise measurements of both their distances (parallaxes) and their absolute PMs are extremely valuable to aid our understanding of their nature and origins.

\section{PROPER MOTIONS AND PARALLAXES}

With just a single star in NGC 5272, there is little more we can do here; however, for the other clusters we can combine the TGAS estimates and uncertainties to calculate weighted average parallaxes and PMs for the clusters. To do this, we perform a Monte-Carlo sampling using the member stars identified in TGAS to estimate the cluster PM and parallax along with their covariance.

We also evaluate the probability of any identified member star being a foreground interloper by calculating the $\chi^{2}$ in

\footnotetext{
${ }^{7}$ The fourth cluster is NGC 6544, which was excluded from our analysis as it has no stars inside $2 R_{\text {tidal }}$ that passed the distance and extinction test in Section 2.2.
}
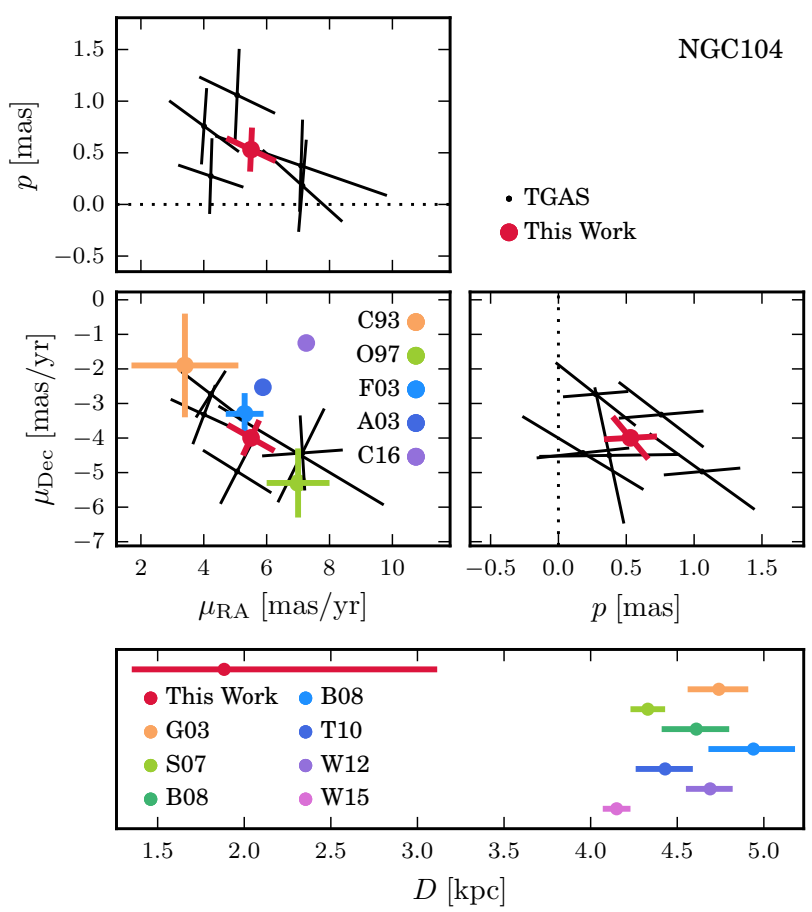

Figure 3. Upper and middle panels: parallax and PM results for NGC 104. The black points show the five TGAS stars that we identified as cluster members. The red points show the mean values that we have calculated here. In the PM plot (middle left) we compare our estimate to values from Cudworth \& Hanson (1993, orange, ground-based), Odenkirchen et al. (1997, green, Hipparcos), Freire et al. (2003, cyan, millisecond pulsars), Anderson \& King (2003, blue, HST), and Cioni et al. (2016, purple, VISTA). Our PM estimate is consistent with both the estimate from MSP timing and the estimate from $H S T$ astrometry. Lower panel: distance comparison for NGC 104. We invert our parallax measurement to obtain a distance estimate (red). We also show previous distance estimates from Gratton et al. (2003, orange, MS fitting), Salaris et al. (2007, light green, HB fitting), Bono et al. (2008, dark green, TRGB; cyan RR Lyraes), Thompson et al. (2010, blue, eclipsing binary), Woodley et al. (2012) (purple, WD SEDs), and Watkins et al. (2015a, pink, dynamical). Our distance is considerably smaller than previous estimates.

parallax for the member stars relative to the literature value, and then the corresponding $p$-value (probability of a $\chi^{2}$ this high or higher occurring by chance). For all clusters, we find $p \gg 0.05$, which indicates that there is no statistical basis to assume that any of the stars in our final samples must be an interloper (although this can never be fully excluded).

All of our results are presented in Table 2 and are compared to results from previous studies in the following subsections. Comparing our parallax estimates to previous measurements is subtly non-trivial, since usually previous studies have estimated distances or distance moduli, so two types of measurements (along with their uncertainties) must be converted into the third type for comparison. The choice of which measurement type to use for comparison is somewhat arbitrary, and we choose to compare distances here, as cluster distances are commonly the quantity that we wish to know.

\subsection{NGC 104 (47 Tucanae)}

For NGC 104, we find parallax $\varpi=0.531 \pm 0.210$ mas, RA $\mathrm{PM} \mu_{\alpha *}=5.50 \pm 0.70$ mas/yr, and Dec PM $\mu_{\delta}=-3.99 \pm$ $0.55 \mathrm{mas} / \mathrm{yr}$, with correlation terms $\rho\left(\varpi, \mu_{\alpha *}\right)=-0.440$, $\rho\left(\varpi, \mu_{\delta}\right)=-0.503$, and $\rho\left(\mu_{\alpha *}, \mu_{\delta}\right)=-0.332$. In Figure 3, the upper and middle panels show the parallaxes and PMs of the five TGAS stars (black) and our averages (red).

There are five previous PM estimates for NGC 104: Cud- 
Table 1

TGAS star properties and membership selection statistics

\begin{tabular}{|c|c|c|c|c|c|c|c|c|c|c|c|c|c|}
\hline GID & 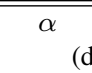 & g) $\delta$ & \multirow{2}{*}{$\begin{array}{c}\varpi \\
\text { (mas) } \\
(4)\end{array}$} & \multicolumn{2}{|c|}{$\begin{array}{c}\mu_{\alpha *} \mu_{\delta} \\
(\mathrm{mas} / \mathrm{yr})\end{array}$} & \multicolumn{2}{|c|}{$\bar{B}_{(\mathrm{mag})} V_{\mathrm{T}}$} & \multirow{2}{*}{$\begin{array}{c}v_{\mathrm{r}} \\
(\mathrm{km} / \mathrm{s}) \\
(9)\end{array}$} & \multirow{2}{*}{$\begin{array}{c}\text { Cluster } \\
\text { (10) }\end{array}$} & \multirow{2}{*}{$\begin{array}{c}\log \mathcal{L}_{\alpha \delta, \mathrm{i}} \\
(11)\end{array}$} & \multirow{2}{*}{$\begin{array}{c}\log \mathcal{L}_{\varpi \mu, \mathrm{i}} \\
(12)\end{array}$} & \multirow{2}{*}{$\begin{array}{c}\log \mathcal{L}_{\mathrm{i}} \\
(13)\end{array}$} & \\
\hline (1) & (2) & (3) & & $(5)$ & (6) & $(7)$ & $(8)$ & & & & & & \\
\hline 4689620330317403136 & 5.91 & -72.28 & 0.274 & 4.22 & -2.73 & 13.82 & 12.01 & $\ldots$ & NGC 104 & -0.204 & -2.478 & -2.683 & $\star$ \\
\hline 4690024022888359424 & 7.30 & -71.82 & 0.109 & 3.96 & -1.60 & 13.64 & 11.72 & -19.28 & NGC 104 & -6.887 & -3.748 & -10.634 & \\
\hline 4689832845301844352 & 6.09 & -71.89 & 1.058 & 5.06 & -4.97 & 14.48 & 11.74 & $\ldots$ & NGC 104 & -0.165 & -4.036 & -4.201 & $\star$ \\
\hline 4689644416501132800 & 6.22 & -71.94 & 0.375 & 7.10 & -4.50 & 13.02 & 12.26 & $\ldots$ & NGC 104 & -0.235 & -3.745 & -3.980 & * \\
\hline 4689638437899435136 & 5.57 & -72.10 & 0.580 & 2.09 & -1.14 & 13.28 & 12.20 & $\ldots$ & NGC 104 & -0.814 & -3.447 & -4.260 & \\
\hline 4689645000616682240 & 6.01 & -71.93 & 0.757 & 4.01 & -3.32 & 13.28 & 11.65 & -30.57 & NGC 104 & -0.093 & -3.006 & -3.099 & * \\
\hline 4689623594492482176 & 6.27 & -72.16 & 0.180 & 7.14 & -4.43 & 12.71 & 11.55 & $\ldots$ & NGC 104 & -0.259 & -4.033 & -4.292 & $\star$ \\
\hline 4689595831823970304 & 5.41 & -72.41 & 0.675 & 4.24 & -3.25 & 13.54 & 12.00 & $\ldots$ & NGC 104 & -1.944 & -3.268 & -5.212 & \\
\hline 1454784965950282240 & 205.46 & 28.39 & 0.225 & -0.69 & -2.85 & 14.74 & 12.46 & -148.86 & NGC 5272 & -0.062 & -0.939 & -1.001 & $\star$ \\
\hline 6045476290889048704 & 245.82 & -26.54 & 0.586 & -12.78 & -18.38 & 14.13 & 11.65 & 69.90 & NGC 6121 & -0.017 & -2.094 & -2.111 & $\star$ \\
\hline 6045462100309586816 & 245.97 & -26.58 & 0.437 & -12.56 & -18.75 & 13.10 & 11.68 & $\ldots$ & NGC 6121 & -0.023 & -2.018 & -2.042 & * \\
\hline 6045462890583403136 & 245.84 & -26.62 & 0.425 & -12.87 & -18.51 & 11.99 & 10.15 & $\ldots$ & NGC 6121 & -0.033 & -1.977 & -2.011 & $\star$ \\
\hline 6045490618893190656 & 246.01 & -26.40 & 0.521 & -13.41 & -18.88 & 12.86 & 10.94 & 66.26 & NGC 6121 & -0.078 & -2.061 & -2.138 & 夫 \\
\hline 6045452204710238080 & 245.86 & -26.79 & 0.810 & -10.95 & -17.14 & 12.93 & 10.91 & $\ldots$ & NGC 6121 & -0.189 & -5.091 & -5.280 & $\star$ \\
\hline 5921742474972627456 & 265.18 & -53.83 & 0.317 & 2.28 & -18.92 & 11.92 & 10.54 & & NGC 6397 & -0.731 & -5.469 & -6.200 & * \\
\hline 5921747938171016320 & 265.08 & -53.70 & -0.150 & 3.45 & -17.91 & 11.79 & 10.18 & $\cdots$ & NGC 6397 & -0.260 & -4.060 & -4.319 & $\star$ \\
\hline 5921745567349079424 & 265.11 & -53.80 & 0.441 & 2.04 & -19.45 & 12.64 & 12.06 & $\ldots$ & NGC 6397 & -0.587 & -5.876 & -6.463 & $\star$ \\
\hline 5921729074674635776 & 265.47 & -53.56 & 0.159 & 3.79 & -17.58 & 12.06 & 10.68 & & NGC 6397 & -2.945 & -7.572 & -10.517 & $\star$ \\
\hline 5921744708355615616 & 265.18 & -53.75 & 0.098 & 4.61 & -17.91 & 11.96 & 10.55 & $\ldots$ & NGC 6397 & -0.148 & -6.352 & -6.500 & $\star$ \\
\hline 5921751030547464960 & 265.24 & -53.66 & -0.041 & 3.14 & -16.24 & 12.41 & 11.20 & 23.21 & NGC 6397 & -0.120 & -8.766 & -8.887 & $\star$ \\
\hline 5921744570916663040 & 265.16 & -53.76 & 0.608 & 1.95 & -17.19 & 11.77 & 10.54 & $\ldots$ & NGC 6397 & -0.202 & -4.154 & -4.357 & $\star$ \\
\hline 4077494753703271424 & 279.04 & -23.92 & 0.585 & 6.98 & -5.99 & 12.31 & 11.09 & -130.00 & NGC 6656 & -0.023 & -4.215 & -4.238 & * \\
\hline 4076740385647365376 & 279.04 & -24.10 & 0.433 & 10.31 & -4.17 & 13.12 & 11.47 & $\ldots$ & NGC 6656 & -0.285 & -5.788 & -6.073 & $\star$ \\
\hline 4077552649862421248 & 278.49 & -23.91 & 0.609 & 8.30 & -1.32 & 12.16 & 12.03 & $\ldots$ & NGC 6656 & -2.650 & -4.648 & -7.298 & \\
\hline 4077590892251201152 & 279.15 & -23.81 & 0.837 & 5.52 & -4.94 & 11.91 & 11.84 & $\ldots$ & NGC 6656 & -0.085 & -4.411 & -4.497 & \\
\hline 4076708431092287104 & 279.21 & -24.53 & 0.533 & 3.28 & -5.00 & 13.84 & 11.39 & $\ldots$ & NGC 6656 & -2.874 & -6.778 & -9.652 & \\
\hline
\end{tabular}

Notes. Columns: (1) Gaia ID; (2) Right Ascension; (3) Declination; (4) parallax; (5) PM in Right Ascension; (6) PM in Declination; (7) Tycho2 B magnitude; (8) Tycho2 V magnitude; (9) radial velocity from RAVE; (10) nearest cluster centre; (11) logarithm of position likelihood; (12) logarithm of parallax and PM likelihood; (13) logarithm of total likelihood. Stars marked $\star$ are those we identify as cluster members and use in Section 3 to derive the cluster properties in Table 2. Uncertainties and correlations are not listed, but are available from the TGAS, Tycho2 and RAVE catalogues.

Table 2

TGAS parallax and proper motion results.

\begin{tabular}{ccccccccccc}
\hline \hline Cluster & $\begin{array}{c}\varpi \\
(\mathrm{mas})\end{array}$ & $\begin{array}{c}\mu_{\alpha *} \\
(\mathrm{mas} / \mathrm{yr})\end{array}$ & $\begin{array}{c}\mu_{\delta} \\
(\mathrm{mas} / \mathrm{yr})\end{array}$ & $\begin{array}{c}\sigma \varpi \\
(\mathrm{mas})\end{array}$ & $\begin{array}{c}\sigma \mu_{\alpha *} \\
(\mathrm{mas} / \mathrm{yr})\end{array}$ & $\begin{array}{c}\sigma \mu_{\delta} \\
(\mathrm{mas} / \mathrm{yr})\end{array}$ & $\rho\left(\varpi, \mu_{\alpha *}\right)$ & $\rho\left(\varpi, \mu_{\delta}\right)$ & $\rho\left(\mu_{\alpha *}, \mu_{\delta}\right)$ & $p_{\mathrm{parallax}}$ \\
\hline NGC 104 & 0.531 & 5.50 & -3.99 & 0.210 & 0.70 & 0.55 & -0.440 & -0.503 & -0.332 & 0.428 \\
NGC 5272 & 0.225 & -0.69 & -2.85 & 0.289 & 0.51 & 0.37 & -0.197 & -0.593 & 0.319 & 0.660 \\
NGC 6121 & 0.556 & -12.51 & -18.33 & 0.149 & 0.50 & 0.29 & 0.589 & 0.519 & 0.488 & 0.930 \\
NGC 6397 & 0.205 & 3.03 & -17.88 & 0.223 & 1.09 & 1.36 & -0.066 & 0.036 & 0.755 & 0.895 \\
NGC 6656 & 0.509 & 8.64 & -5.09 & 0.222 & 1.49 & 1.45 & -0.194 & -0.160 & 0.935 & 0.630 \\
\hline
\end{tabular}

Notes. Columns: (1) cluster name; (2) parallax estimate; (3) RA PM estimate; (4) Dec PM estimate; (5) uncertainty on parallax estimate; (6) uncertainty on RA PM estimate; (7) uncertainty on Dec PM estimate; (8) correlation between parallax and RA PM estimates; (9) correlation between parallax and Dec PM estimates; (10) correlation between RA PM and Dec PM estimates; (11) $p$-value for parallax.

worth \& Hanson (1993) measured $\mu_{\alpha *}=3.4 \pm 1.7$ mas/yr and $\mu_{\delta}=-1.9 \pm 1.5 \mathrm{mas} / \mathrm{yr}$ from the ground; Odenkirchen et al. (1997) measured $\mu_{\alpha *}=7.0 \pm 1.0 \mathrm{mas} / \mathrm{yr}$ and $\mu_{\delta}=$ $-5.3 \pm 1.0 \mathrm{mas} / \mathrm{yr}$ using Hipparcos data; Freire et al. (2003) measured $\mu_{\alpha *}=5.3 \pm 0.6 \mathrm{mas} / \mathrm{yr}$ and $\mu_{\delta}=-3.3 \pm 0.6 \mathrm{mas} / \mathrm{yr}$ using radio observations of millisecond pulsars (MSPs); Anderson \& King (2003) measured $\mu_{\alpha *}=5.88 \pm 0.18 \mathrm{mas} / \mathrm{yr}$ and $\mu_{\delta}=-2.53 \pm 0.18 \mathrm{mas} / \mathrm{yr}$ using HST/WFPC2 ${ }^{8}$; and

\footnotetext{
8 We did not use the absolute PM given in Anderson \& King (2003); instead we used their measured motion of NGC 104 relative to the Small Magellanic Cloud (SMC) combined with the SMC PM measurement from Kalli-
}

Cioni et al. (2016) measured $\mu_{\alpha *}=7.26 \pm 0.03 \mathrm{mas} / \mathrm{yr}$ and $\mu_{\delta}=-1.25 \pm 0.03 \mathrm{mas} / \mathrm{yr}$ using ground-based data from VISTA. These points are shown in the PM panel (centre left) of Figure 3 as orange, green, cyan, blue, and purple points, respectively.

Our PM estimate shows best agreement with the estimate derived from MSP timing by Freire et al. (2003) and the HST measurement by Anderson \& King (2003); for the latter, our RA PM is in very good agreement, though are Dec PM is slightly larger than theirs.

vayalil et al. (2006). 
Our parallax of $\varpi=0.531 \pm 0.210$ mas corresponds to a distance of $D=1.88_{-0.53}^{+1.23} \mathrm{kpc}$. NGC 104 is one of the best studied Galactic GCs and so there are many estimates of its distance in the literature; for a complete overview of previous distance determinations, see Table 2 of Woodley et al. (2012). Here we compare with only a subset of these chosen to cover a wide variety of estimation methods: Gratton et al. (2003) estimated $D=4.74_{-0.17}^{+0.18} \mathrm{kpc}$ via MS fitting; Salaris et al. (2007) estimated $D=4.33 \pm 0.10 \mathrm{kpc}$ via HB fitting; Bono et al. (2008) estimated $D=4.61_{-0.19}^{+0.20} \mathrm{kpc}$ using the tip of the Red Giant Branch (TRGB) and $D=4.94_{-0.24}^{+0.26} \mathrm{kpc}$ using RR Lyraes; Thompson et al. (2010) estimated $D=4.43_{-0.16}^{+0.17} \mathrm{kpc}$ using an eclipsing binary; Woodley et al. (2012) estimated $D=4.69_{-0.13}^{+0.14} \mathrm{kpc}$ using white dwarf (WD) spectral energy distributions (SEDs); and Watkins et al. (2015a) used estimated $D=4.15 \pm 0.08 \mathrm{kpc}$ using stellar kinematics. We show these distance estimates along with our own in the lower panel of Figure 3. Our distance estimate is considerably smaller than all of the previous distance estimates for NGC 104.

NGC 104 is the only cluster of the five in our sample for which the TGAS distance estimate is not consistent at $1 \sigma$ with literature estimates. It is is possible that this reflects some level of foreground contamination in our sample of five stars. ${ }^{9}$ Three of the stars have individual parallax estimates consistent with the known NGC 104 distance, while the other two do not (see Figure 3, top-left and middle-right panels).

\subsection{NGC $5272(M 3)$}

For NGC 5272, we have only one member star, so we do not perform a Monte-Carlo sampling but instead report the TGAS values for the single member star. The TGAS estimate finds parallax $\varpi=0.225 \pm 0.289$ mas, RA PM $\mu_{\alpha *}=-0.69 \pm$ $0.51 \mathrm{mas} / \mathrm{yr}$, and Dec PM $\mu_{\delta}=-2.85 \pm 0.37$ mas/yr, with correlation terms $\rho\left(\varpi, \mu_{\alpha *}\right)=-0.197, \rho\left(\varpi, \mu_{\delta}\right)=-0.593$, and $\rho\left(\mu_{\alpha *}, \mu_{\delta}\right)=0.319$. In Figure 4, the upper and middle panels show the parallax and PMs of the identified TGAS star.

There are five previous PM estimates for NGC 5272; unfortunately there are no HST measurements for comparison, all are ground-based estimates and they are as follows. Cudworth \& Hanson (1993) measured $\mu_{\alpha *}=-1.2 \pm 2.5 \mathrm{mas} / \mathrm{yr}$ and $\mu_{\delta}=2.4 \pm 3.0 \mathrm{mas} / \mathrm{yr}$ from the ground; Scholz et al. (1993) measured $\mu_{\alpha *}=-3.1 \pm 0.2 \mathrm{mas} / \mathrm{yr}$ and $\mu_{\delta}=$ $-2.3 \pm 0.4 \mathrm{mas} / \mathrm{yr}$ using photographic plates; Odenkirchen et al. (1997) measured $\mu_{\alpha *}=0.9 \pm 1.0 \mathrm{mas} / \mathrm{yr}$ and $\mu_{\delta}=$ $-2.2 \pm 1.0 \mathrm{mas} / \mathrm{yr}$ using Hipparcos data; Geffert (1998) measured $\mu_{\alpha *}=-1.2 \pm 0.8 \mathrm{mas} / \mathrm{yr}$ and $\mu_{\delta}=-3.2 \pm 0.8 \mathrm{mas} / \mathrm{yr}$ also using Hipparcos stars; and Wu et al. (2002) measured $\mu_{\alpha *}=-0.06 \pm 0.3 \mathrm{mas} / \mathrm{yr}$ and $\mu_{\delta}=-2.6 \pm 0.3 \mathrm{mas} / \mathrm{yr}$ using photographic plates spanning a 70 year baseline. The latter four points are shown in the PM panel (centre left) of Figure 4 as orange, green, cyan, and blue points, respectively. The estimate by Cudworth \& Hanson (1993) is not shown as it is significantly offset from the others and is not located within the plot limits. The TGAS PM estimate shows best agreement with the estimate derived using photographic plates by Wu et al. (2002) and the Hipparcos measurement by Geffert (1998).

Our parallax of $\varpi=0.225 \pm 0.289$ mas corresponds to a distance of $D=4.44_{-2.50}^{+\infty} \mathrm{kpc}$. The upper error bar on the

\footnotetext{
9 Though, as noted above, we find a $p \gg 0.05$ for the cluster, so it is statistical unlikely that any interlopers remain.
}
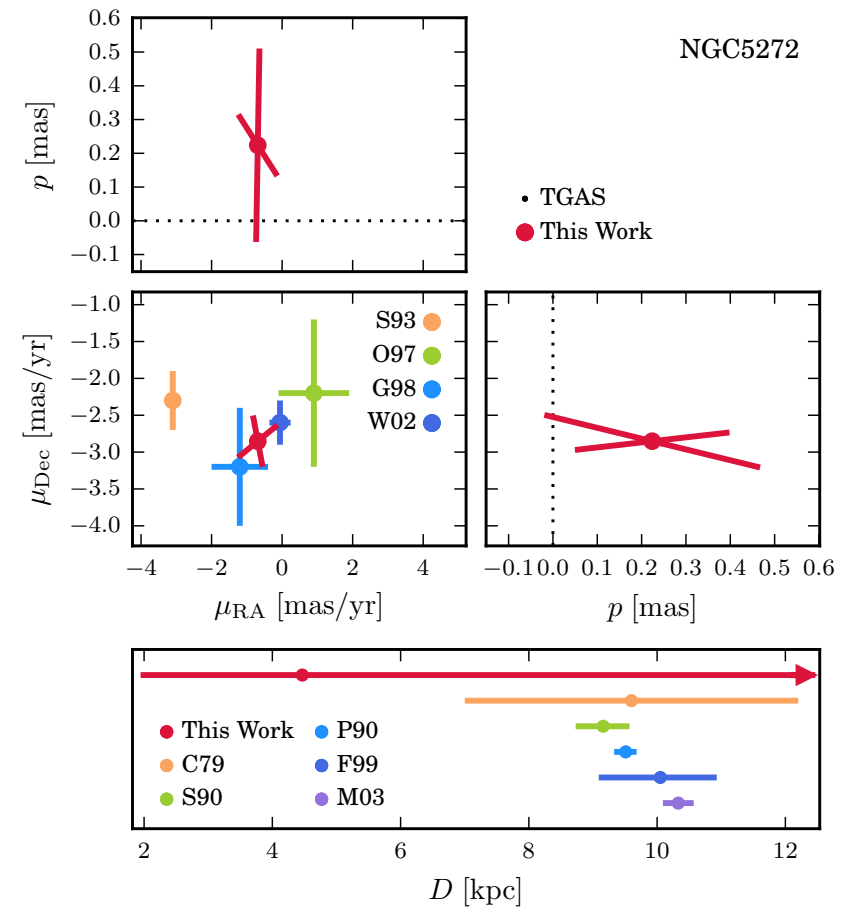

Figure 4. Upper and middle panels: parallax and PM results for NGC 5272. The black points show the TGAS star that we identified as a cluster member. In the PM plot (middle left) we compare our estimate to values from Scholz et al. (1993, orange, photographic plates), Odenkirchen et al. (1997, green, Hipparcos), Geffert (1998, cyan, Hipparcos), and Wu et al. (2002, blue, photographic plates). Our PM estimate is consistent with both of the latter two estimates. (There is a further estimate given given by Cudworth \& Hanson (1993), however, this is not shown as it does not lie within the plot limits.) Lower panel: distance comparison for NGC 5272. We invert our parallax measurement to obtain a distance estimate (red). We also show previous distance estimates from Cudworth (1979, orange, dynamical), Sandage \& Cacciari (1990, green, MS fitting), Paez et al. (1990, cyan, CMD fitting), Ferraro et al. (1999, blue, HB fitting), and Marconi et al. (2003, purple, RR Lyrae pulsations). Our distance is considerably smaller than previous estimates.

distance is unconstrained, since the lower error bar on the parallax extends below zero, which, as previously discussed, is unphysical but statistically rigourous. NGC 5272 has a number of previous estimates of its distance in the literature; here we compare with only a subset of these chosen to cover a wide variety of estimation methods: Cudworth (1979) estimated $D=9.6 \pm 2.6 \mathrm{kpc}$ from cluster kinematics; Sandage \& Cacciari (1990) estimated $D=9.16_{-0.41}^{+0.43} \mathrm{kpc}$ via MS fitting; Paez et al. (1990) estimated $D=9.51_{-0.17}^{+0.18} \mathrm{kpc}$ using colourmagnitude diagram (CMD) fitting; Ferraro et al. (1999) estimated $D=10.05_{-0.88}^{+0.97} \mathrm{kpc}$ using HB fitting; and Marconi et al. (2003) estimated $D=10.33 \pm 0.24 \mathrm{kpc}$ using RR Lyrae pulsations. We show these distance estimates along with our own in the lower panel of Figure 4. Our distance estimate is considerably smaller than all of the previous distance estimates for NGC 5272.

\section{3. $N G C 6121(M 4)$}

For NGC 6121, we find parallax $\varpi=0.556 \pm 0.149$ mas, RA PM $\mu_{\alpha *}=-12.51 \pm 0.50 \mathrm{mas} / \mathrm{yr}$, and Dec PM $\mu_{\delta}=$ $-18.33 \pm 0.29 \mathrm{mas} / \mathrm{yr}$, with correlation terms $\rho\left(\varpi, \mu_{\alpha *}\right)=$ $0.589, \rho\left(\varpi, \mu_{\delta}\right)=0.519$, and $\rho\left(\mu_{\alpha *}, \mu_{\delta}\right)=0.488$. In Figure 5 , the upper and middle panels show the parallaxes and PMs of the 5 TGAS stars (black) and our averages (red).

To define membership probabilities in Section 2.3, we used the PM from CD13 as a reference. In fact, the PM 


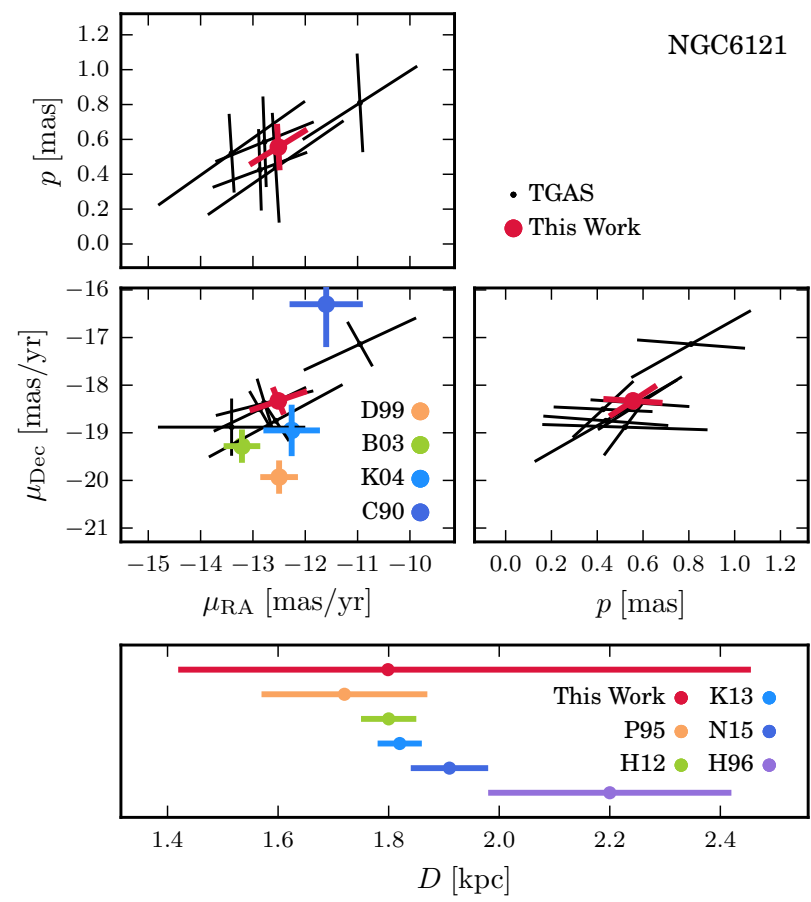

Figure 5. Upper and middle panels: parallax and PM results for NGC 6121. The black points show the five TGAS stars that we identified as cluster members. The red points show the mean values we have calculated here. In the PM plot (middle left) we compare our estimate to values from Dinescu et al. (1999a, orange, ground-based), Bedin et al. (2003, green, HST), Kalirai et al. (2004, cyan, HST), and Cudworth \& Rees (1990, blue, ground-based). The PMs we measure are consistent with the previous HST measurements and have similar uncertainties. Lower panel: distance comparison for NGC 6121. We invert our parallax measurement to obtain a distance estimate (red). We also show previous distance estimates from Peterson et al. (1995, orange, dynamical), Hendricks et al. (2012, green, HB luminosity), Kaluzny et al. (2013, cyan, eclipsing binaries), Neeley et al. (2015, blue, RR Lyraes), and the estimate from H96 (purple). Our distance is consistent with previous estimates within our uncertainties, but our uncertainties are not yet competitive with existing results.

for NGC 6121 given in that catalogue is a combination of three separate studies and so we compare our PM again each of those studies here: Dinescu et al. (1999a) measured $\mu_{\alpha *}=-12.50 \pm 0.36 \mathrm{mas} / \mathrm{yr}$ and $\mu_{\delta}=-19.93 \pm$ 0.35 mas/yr from SPM photographic plates using Hipparcos stars as a reference frame; Bedin et al. (2003) measured $\mu_{\alpha *}=-13.21 \pm 0.35 \mathrm{mas} / \mathrm{yr}$ and $\mu_{\delta}=-19.28 \pm 0.35 \mathrm{mas} / \mathrm{yr}$ using HST/WFPC2 data; and Kalirai et al. (2004) measured $\mu_{\alpha *}=-12.26 \pm 0.54 \mathrm{mas} / \mathrm{yr}$ and $\mu_{\delta}=-18.95 \pm 0.54 \mathrm{mas} / \mathrm{yr}$ also using HST/WFPC2 but with a longer baseline. These points are shown in the PM panel (centre left) of Figure 5 as orange, green, and cyan points, respectively.

Our PM in RA is consistent with all three studies, but our PM in Dec is in much better agreement with the two HST PMs than the ground-based PM. HST tends to be less susceptible to various kinds of errors than ground-based measurements, so the good agreement between the HST and TGAS estimates is very encouraging. This is also consistent with the conclusions from van der Marel \& Sahlmann (2016) who recently studied rotation in the Large and Small Magellanic Clouds using TGAS and found excellent agreement between the TGAS estimates and earlier HST studies.

There is one further estimate of the PM of NGC 6121 that used bright field stars to create a reference frame to measure $\mu_{\alpha *}=-11.6 \pm 0.7 \mathrm{mas} / \mathrm{yr}$ and $\mu_{\delta}=-16.3 \pm 0.9 \mathrm{mas} / \mathrm{yr}$ (Cudworth \& Rees 1990, shown in blue), which is signif- icantly offset from the other previous studies and our own TGAS estimate.

Our parallax of $\varpi=0.556 \pm 0.149$ mas corresponds to a distance of $D=1.80_{-0.38}^{+0.66} \mathrm{kpc}$. This is in good agreement with the distance of $D=2.2 \mathrm{kpc}$ from H96, which we inverted to obtain a parallax for the membership probability analysis in Section 2.3. As the closest GC, M4 has been well studied, so there are many estimates of its distance in the literature, and in fact the H96 value is among the largest. We summarise a selection here covering a wide variety of estimation methods: Peterson et al. (1995) used PMs and LOS velocity dispersions to estimate a distance of $D=1.72 \pm 0.14 \mathrm{kpc}$; Hendricks et al. (2012) used the luminosity of the HB to measure $D=1.80 \pm 0.05 \mathrm{kpc}$; Kaluzny et al. (2013) used three eclipsing binaries in NGC 6121 to measure $D=1.82 \pm 0.04 \mathrm{kpc}$; and Neeley et al. (2015) used RR Lyraes to measure $D=1.91 \pm 0.07 \mathrm{kpc}$. We show these distance estimates along with our own in the lower panel of Figure 5.

Our distance estimate is consistent with all previous estimates within their uncertainties, however, our distance uncertainty is considerably larger than the previous results.

\subsection{NGC 6397}

For NGC 6397, we find parallax $\varpi=0.205 \pm 0.223$ mas, RA PM $\mu_{\alpha *}=3.03 \pm 1.09$ mas $/ y r$, and Dec PM $\mu_{\delta}=$ $-17.88 \pm 1.36 \mathrm{mas} / \mathrm{yr}$, with correlation terms $\rho\left(\varpi, \mu_{\alpha *}\right)=$ $-0.066, \rho\left(\varpi, \mu_{\delta}\right)=0.036$, and $\rho\left(\mu_{\alpha *}, \mu_{\delta}\right)=0.755$. In Figure 6, the upper and middle panels show the parallaxes and PMs of the seven TGAS stars (black) and our averages (red).

There are four previous PM estimates for NGC 6397: Cudworth \& Hanson (1993) measured $\mu_{\alpha *}=3.3 \pm 0.5$ mas $/ \mathrm{yr}$ and $\mu_{\delta}=-15.2 \pm 0.6 \mathrm{mas} / \mathrm{yr}$ from the ground; Milone et al. (2006) measured $\mu_{\alpha *}=3.39 \pm 0.15 \mathrm{mas} / \mathrm{yr}$ and $\mu_{\delta}=-17.55 \pm 0.15$ mas/yr using HST/WFPC2 data; Kalirai et al. (2007) measured $\mu_{\alpha *}=3.56 \pm 0.04$ mas/yr and $\mu_{\delta}=$ $-17.34 \pm 0.04$ mas/yr using a combination of HST/WFPC2 and HST/ACS data; and CD13 measured $\mu_{\alpha *}=3.69 \pm$ $0.29 \mathrm{mas} / \mathrm{yr}$ and $\mu_{\delta}=-14.88 \pm 0.26 \mathrm{mas} / \mathrm{yr}$ using a combination of photographic plates and ground-based CCD images. These points are shown in the PM panel (centre left) of Figure 6 as orange, green, cyan, and blue points, respectively. Note that all of these measurements are in reasonable agreement in $\mu_{\alpha *}$, but are discrepant at $\sim 2$ mas/yr in $\mu_{\delta}$. We find that our $\mu_{\delta}$ estimate is in much better agreement with the two HST PMs than the two ground-based PMs. This trend is consistent with our findings for NGC 6121 in Section 3.3.

Our parallax of $\varpi=0.205 \pm 0.223$ mas corresponds to a distance of $D=4.88_{-2.55}^{+\infty} \mathrm{kpc}$. Our upper error bar on the distance is unconstrained, since the lower error bar on the parallax for NGC 6397 extends below zero, which, as previously discussed, is unphysical but statistically rigourous.

There are a number of previous distance estimates in the literature, of which we show a representative sample here, obtained using different methods: Reid \& Gizis (1998) estimated $D=2.67_{-0.18}^{+0.19} \mathrm{kpc}$ and Gratton et al. (2003) estimated $D=2.54_{-0.09}^{+0.10} \mathrm{kpc}$, both via MS fitting; Hansen et al. (2007) measured $D=2.54 \pm 0.07 \mathrm{kpc}$ by fitting the WD sequence; and Heyl et al. (2012) estimated $D=2.2_{-0.7}^{+0.5} \mathrm{kpc}$ and Watkins et al. (2015a) estimated $D=2.39_{-0.11}^{+0.13} \mathrm{kpc}$ by combining PMs and LOS velocities. We show these distance estimates along with our own in the lower panel of Figure 6. Our result is larger than all of these previous estimates, but is 


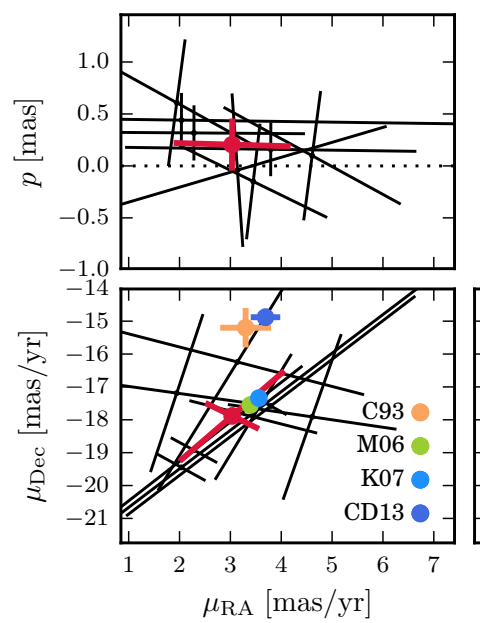

- TGAS

This Work
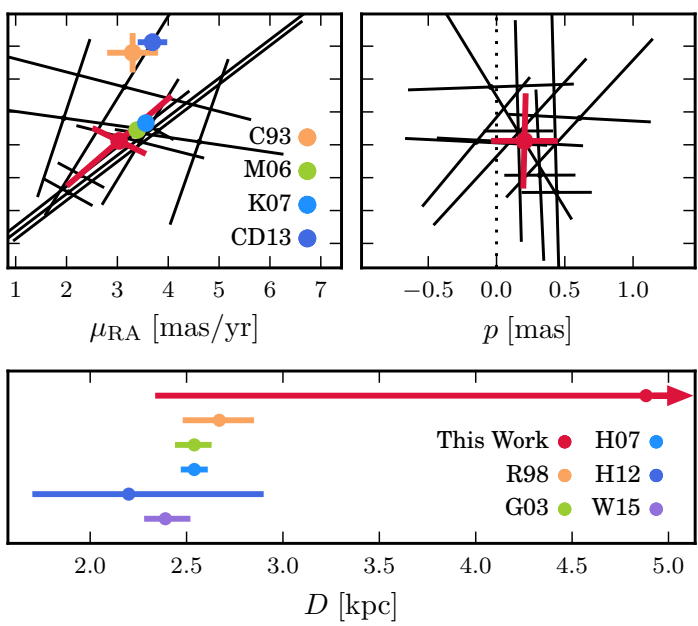

Figure 6. Upper and middle panels: parallax and PM results for NGC 6397. The black points show the seven TGAS stars that we identified as cluster members. The red points show the mean values we have calculated here. In the PM plot (middle left) we compare our estimate to values from Cudworth \& Hanson (1993, orange, ground-based), Milone et al. (2006, green, HST), Kalirai et al. (2007, cyan, HST), and CD13 (blue, ground-based). The PMs we measure are consistent with the previous HST measurements, albeit with uncertainties an order of magnitude larger and significantly offset from the previous ground-based measurements. Lower panel: distance comparison for NGC 6397. We invert our parallax measurement to obtain a distance estimate (red); no upper error bar is shown, since lower error bar on the parallax extended below zero, corresponding to unphysical results but effectively indicating that the upper limit on the distance uncertainty is infinite. We also show previous distance estimates from Reid \& Gizis (1998, orange, MS fitting), Gratton et al. (2003, green, MS fitting), Hansen et al. (2007, cyan, WD sequence), Heyl et al. (2012, blue, dynamical), and the estimate from Watkins et al. (2015a, purple, dynamical). Our distance uncertainties are significantly larger than previous estimates, however, our distance estimate is consistent within the previous results within the very large error bars.

consistent within $1 \sigma$.

\subsection{NGC 6656 (M 22)}

For NGC 6656, we find parallax $\varpi=0.509 \pm 0.222$ mas, RA PM $\mu_{\alpha *}=8.64 \pm 1.49 \mathrm{mas} / \mathrm{yr}$, and Dec PM $\mu_{\delta}=$ $-5.09 \pm 1.45 \mathrm{mas} / \mathrm{yr}$, with correlation terms $\rho\left(\varpi, \mu_{\alpha *}\right)=$ $-0.194, \rho\left(\varpi, \mu_{\delta}\right)=-0.160$, and $\rho\left(\mu_{\alpha *}, \mu_{\delta}\right)=0.935$. In Figure 7, the upper and middle panels show the parallaxes and PMs of the two TGAS stars (black) and our averages (red).

There are three previous PM estimates for NGC 6656: Cudworth \& Hanson (1993) measured $\mu_{\alpha *}=8.6 \pm 1.3$ mas/yr and $\mu_{\delta}=-5.1 \pm 1.5 \mathrm{mas} / \mathrm{yr}$ from the ground; Chen et al. (2004) measured $\mu_{\alpha *}=10.19 \pm 0.20 \mathrm{mas} / \mathrm{yr}$ and $\mu_{\delta}=-3.34 \pm$ $0.10 \mathrm{mas} / \mathrm{yr}$ using HST/WFPC2 data; and CD13 measured $\mu_{\alpha *}=7.37 \pm 0.50 \mathrm{mas} / \mathrm{yr}$ and $\mu_{\delta}=-3.95 \pm 0.42 \mathrm{mas} / \mathrm{yr}$ using a combination of photographic plates and ground-based CCD images. These points are shown in the PM panel (centre left) of Figure 7 as orange, green, and cyan points, respectively. CD13 advises caution considering the Chen et al. (2004) HST result, as they note that bulge stars were used to create the reference frame and that the possible (and likely) motion of the bulge stars was not factored into the analysis,
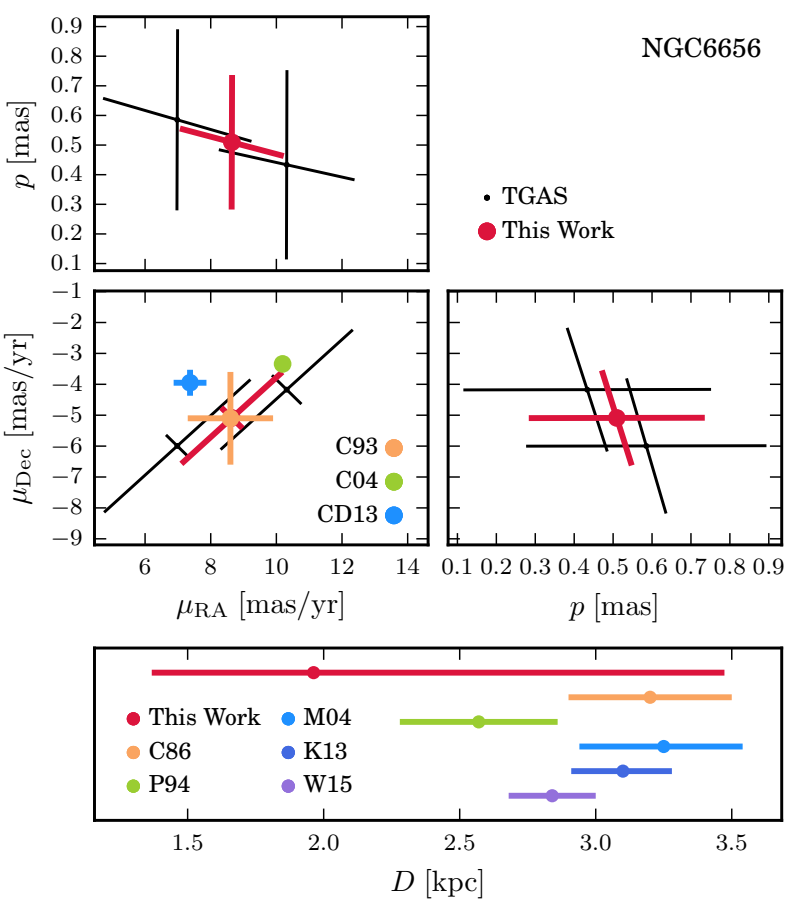

Figure 7. Upper and middle panels: parallax and PM results for NGC 6656. The black points show the two TGAS stars that we identified as cluster members. The red points show the mean values we have calculated here. In the PM plot (middle left) we compare our estimate to values from Cudworth \& Hanson (1993, orange, ground-based), Chen et al. (2004, green, HST), and CD13 (cyan, ground-based). The PMs we measure are consistent with the previous measurements, albeit with uncertainties an order of magnitude larger. Lower panel: distance comparison for NGC 6656. We invert our parallax measurement to obtain a distance estimate (red). We also show previous distance estimates from Cudworth (1986, orange, HB fitting), Peterson \& Cudworth (1994, green, dynamical), Monaco et al. (2004, cyan, HB fitting), Kunder et al. (2013, blue, RR Lyraes), and Watkins et al. (2015a, purple, dynamical). Our distance uncertainties are significantly larger than previous estimates, however, our distance estimate is consistent within the previous results within the our uncertainties.

nevertheless we do make a comparison here.

We find that our PM estimate is consistent with all three studies, indeed it is in exceptional agreement with the groundbased PM estimate from Cudworth \& Hanson (1993). Contrary to the two previous clusters, we do not find the best agreement with the previous $H S T$ estimate, possibly for the reasons discussed above.

Our parallax of $\varpi=0.509 \pm 0.222$ mas corresponds to a distance of $D=1.96_{-0.59}^{+1.51} \mathrm{kpc}$. There are a number of previous distance estimates in the literature obtained using different methods: Cudworth (1986) estimated $D=3.2 \pm 0.3 \mathrm{kpc}$ (which is the value quoted in H96) and Monaco et al. (2004) estimated $D=3.25_{-0.29}^{+0.31} \mathrm{kpc}$, both via HB fitting; Peterson \& Cudworth (1994) measured $D=2.57 \pm 0.29 \mathrm{kpc}$ and Watkins et al. (2015a) estimated $D=2.84 \pm 0.16 \mathrm{kpc}$ by combining PMs and LOS velocities; and Kunder et al. (2013) estimated $D=3.10_{-0.18}^{+0.19} \mathrm{kpc}$ using RR Lyraes. We show these distance estimates along with our own in the lower panel of Figure 7. This time, our result is lower than all of the previous estimates, but is consistent within the error bars. Once again, our distance uncertainties are an order of magnitude larger than for previous estimates.

\section{ABSOLUTE SPACE MOTIONS}


Table 3

Additional cluster properties for space motion analysis.

\begin{tabular}{cccc}
\hline \hline Cluster & $\begin{array}{c}\alpha \\
(\mathrm{deg})\end{array}$ & $\begin{array}{c}\delta \\
(\mathrm{deg})\end{array}$ & $\begin{array}{c}D \\
(\mathrm{kpc}) \\
(1)\end{array}$ \\
$(2)$ & $(3)$ & $(4)$ \\
\hline NGC 104 & 6.024 & -72.081 & $4.61_{-0.19}^{+0.20}$ \\
NGC 5272 & 205.548 & 28.377 & $10.05_{-0.88}^{+0.97}$ \\
NGC 6121 & 245.897 & -26.526 & $1.80 \pm 0.05$ \\
NGC 6397 & 265.175 & -53.674 & $2.54_{-0.09}^{+0.10}$ \\
NGC 6656 & 279.100 & -23.905 & $3.10_{-0.18}^{+0.19}$ \\
\hline
\end{tabular}

Notes. Columns: (1) cluster name; (2) Right Ascension; (3) Declination; (4) adopted distance (sources: Bono et al. (2008, NGC 104), Ferraro et al. (1999, NGC 5272), Hendricks et al. (2012, NGC 6121), Gratton et al. (2003, NGC 6397), and Kunder et al. (2013, NGC 6656)).

We can combine our PM estimates with sky coordinates, distances, and heliocentric RVs to determine the implied Galactocentric motion of the GCs. We use our PM estimates for this analysis, but not our distance (parallax) estimates, as it is clear those are less precise than literature values. The sky coordinates and distances we used here are listed in Table 3.

We adopt a Cartesian coordinate system $(X, Y, Z)$ centred on the Galactic Centre, where the $X$-axis points in the direction from the Sun to the Galactic Centre, the $Y$-axis points in the direction of the Sun's Galactic rotation, and the $Z$-axis points toward the North Galactic Pole.

To transform the measured heliocentric velocities into velocities $(U, V, W)$ in the Galactocentric rest frame, we adopt a distance from the Sun to the Galactic Centre of $R_{0}=$ $8.29 \pm 0.16 \mathrm{kpc}$, and a circular velocity of the local standard of rest (LSR) of $V_{0}=239 \pm 5 \mathrm{~km} / \mathrm{s}$ (both McMillan 2011). We also assume a solar peculiar velocity relative to the LSR of $\left(U_{\text {pec }}, V_{\text {pec }}, W_{\text {pec }}\right)=(11.10 \pm 1.23,12.24 \pm 2.05,7.25 \pm$ $0.63) \mathrm{km} / \mathrm{s}$ (Schönrich et al. 2010).

To estimate Galactocentric velocities and their uncertainties, we use a Monte-Carlo scheme that propagates all observational distance and velocity uncertainties and their correlations, including those for the Sun. We also provide Galactocentric radial $v_{\text {rad }}$ and tangential $v_{\text {tan }}$ velocities defined in a spherical coordinate system, where $v_{\text {rad }}$ is positive outward from the Galactic Centre and $v_{\text {tan }}$ is the magnitude of the motion perpendicular to the radial motion. We also calculate the radial $\Pi$ and tangential $\Theta$ velocities in a cylindrical coordinate system, where $\Pi$ is again positive outward from the Galactic Centre and $\Theta$ is positive in the direction of Galactic rotation. Our results are summarised in Table 4.

Zinn (1985) separated Galactic GCs into two subpopulations based on their metallicities and orbital properties: those belonging to the disk $([\mathrm{Fe} / \mathrm{H}]>-0.8)$ and those belonging to the halo $([\mathrm{Fe} / \mathrm{H}]<-0.8)$. Overall, the disk clusters have a lower orbital eccentricity and are confined to the Galactic plane, while the halo clusters span a range of eccentricities and explore greater distances from the disk plane.

In their orbital analysis, Odenkirchen et al. (1997) noted that the orbital energy of NGC 104 is very close to its corresponding circular orbit (ie. the energy of a circular orbit at its current Galactocentric distance). They noted that the orbital properties of NGC 104 were somewhat offset from the rest of their sample, which were all thought to be halo clusters, and so concluded that NGC 104 is a disk cluster. This conclusion was also reached by Cudworth \& Hanson (1993), who noted that the space velocity of NGC 104 marks it as a thick-disk cluster, despite its relatively large distance from the plane.

NGC 5272 is currently located almost $10 \mathrm{kpc}$ above the plane of the disk, so it is extremely likely to be a halo cluster. All five of the previous PM studies discussed in Section 3.2 performed space motion analyses. Cudworth \& Hanson (1993) found that the orbit of NGC 5272 was very uncertain in their analysis; nevertheless, they concluded that the orbit was more consistent with the Zinn (1985) halo population, as expected. Their space motion was somewhat different from the other studies, but all of the other studies reached broadly similar conclusions: NGC 5272 is a halo cluster with a boxlike orbit (Scholz et al. 1993; Wu et al. 2002) and is likely near its apocentre (Geffert 1998).

NGC 6121 is located very close to the Galactic plane and has a very small $Z$ component of velocity, which implies that it spends most of its time in the disk. However, both Dinescu et al. (1999a) and Bedin et al. (2003) found very radial orbits for NGC 6121, and their extreme eccentricities favour NGC 6121 as being a halo cluster on a very low inclination orbit and not a disk cluster. The PM determination of Cudworth \& Hanson (1993) was significantly offset from all other previous estimates, however, their conclusions on the orbit of NGC 6121 were nonetheless broadly similar.

Our space motion for NGC 6121 is in reasonable agreement with the analyses by Dinescu et al. (1999a) and Bedin et al. (2003), but shows best agreement with that calculated by Kalirai et al. (2004) - which is to be expected given the very good agreement between our PM determination and theirs except for the sign of $W$. This sign discrepancy is not surprising, as we find a very small $W$ component that is consistent with both positive and negative $W$ velocities within $1 \sigma$. Small changes in the assumed parameters will change the sign of the $W$ velocity, but this has little effect on our overall conclusions.

Dinescu et al. (1999a) also estimated the tidal-shock rate of NGC 6121 based on its orbit and found that this is larger than the two-body relaxation time in the cluster; this indicates that tidal shocks due to the bulge and the disk have likely had a significant influence on the evolution of the cluster. Again, it is not surprising that a cluster confined to the disk should be strongly affected by tides.

NGC 6397 is also currently located close to the disk plane, however, the high $\mathrm{W}$ velocity implies that it does move away from the plane and is most likely a halo cluster, as expected from its low $[\mathrm{Fe} / \mathrm{H}]$ metallicity. This is consistent with more detailed orbital analyses by Cudworth \& Hanson (1993) and CD13 which concluded that NGC 6397 has a "typical halolike orbit" (following Zinn 1985). Milone et al. (2006) found that NGC 6397 has a boxy orbit that oscillates rapidly through the disk. Thanks to the moderate eccentricity of the orbit, NGC 6397 does not move too far from the Galactic centre; thus, it passes through high-density regions of the disk and is expected to be affected by tidal shocks.

Similarly, NGC 6656 is also currently located close to the disk plane but its $W$ velocity suggests that it does move away from the disk, so it is more likely to be a halo cluster. From detailed studies of the implied orbit, both Cudworth \& Hanson (1993) and CD13 also concluded that NGC 6656 is a halo cluster. This is not surprising given that NGC 6656 is metalpoor and also shows signs of inhomogeneous element abundances (e.g., Norris \& Freeman 1983), which is thought to indicate extended star formation (e.g., Marino et al. 2011) and point to an extragalactic origin (e.g., Lee et al. 2009). NGC 6656 is also likely to be vulnerable to tides from re- 
Table 4

Cluster space motions and orbits.

\begin{tabular}{|c|c|c|c|c|c|c|c|c|c|c|c|}
\hline Cluster & $\begin{array}{c}X \\
(\mathrm{kpc}) \\
(2)\end{array}$ & $\begin{array}{c}Y \\
(\mathrm{kpc}) \\
(3)\end{array}$ & $\begin{array}{c}Z \\
(\mathrm{kpc}) \\
(4)\end{array}$ & $\begin{array}{c}U \\
(\mathrm{~km} / \mathrm{s}) \\
(5)\end{array}$ & $\begin{array}{c}V \\
(\mathrm{~km} / \mathrm{s}) \\
(6)\end{array}$ & $\begin{array}{c}W \\
(\mathrm{~km} / \mathrm{s}) \\
(7)\end{array}$ & $\begin{array}{c}\mathbf{v} \mid \\
(\mathrm{km} / \mathrm{s}) \\
(8)\end{array}$ & $\begin{array}{c}v_{\mathrm{rad}} \\
(\mathrm{km} / \mathrm{s}) \\
(9)\end{array}$ & $\begin{array}{c}v_{\tan } \\
(\mathrm{km} / \mathrm{s}) \\
(10)\end{array}$ & $\begin{array}{c}\Pi \\
(\mathrm{km} / \mathrm{s}) \\
(11) \\
\end{array}$ & $\begin{array}{c}\Theta \\
(\mathrm{km} / \mathrm{s}) \\
(12) \\
\end{array}$ \\
\hline NGC 1 & -6.38 & -2.65 & -3.25 & $-74.97 \pm 1$ & $5.23 \pm 1$ & $30 \pm$ & $4 \pm 6.81$ & .81 & .02 & 20 & 51 \\
\hline NGC 61 & $1-6.58$ & -0.27 & 0.50 & $55.10 \pm 2.02$ & $50.19 \pm 8.42$ & $2.13 \pm 3.97$ & $74.57 \pm 6.38$ & $-56.81 \pm 2.35$ & $48.30 \pm 8.31$ & $-57.07 \pm 2.16$ & $47.87 \pm 8.47$ \\
\hline NGC 6397 & $7-5.98$ & -0.92 & -0.53 & $-61.20 \pm 7.73$ & $98.79 \pm 16.28$ & $-132.62 \pm 14.60$ & $176.34 \pm 12.75$ & $56.72 \pm 10.39$ & $166.97 \pm 13.49$ & $45.52 \pm 9.48$ & $106.88 \pm 14.93$ \\
\hline
\end{tabular}

Notes. Columns: (1) cluster name; (2) $X$ position coordinate; (3) $Y$ position coordinate; (4) $Z$ position coordinate; (5) $U$ velocity component; (6) $V$ velocity component; (7) $W$ velocity component; (8) magnitude of total velocity; (9) radial component of velocity in a spherical coordinate system; (10) tangential component of velocity in a spherical coordinate system; (11) radial component of velocity in a cylindrical coordinate system; (12) tangential component of velocity in a cylindrical coordinate system.

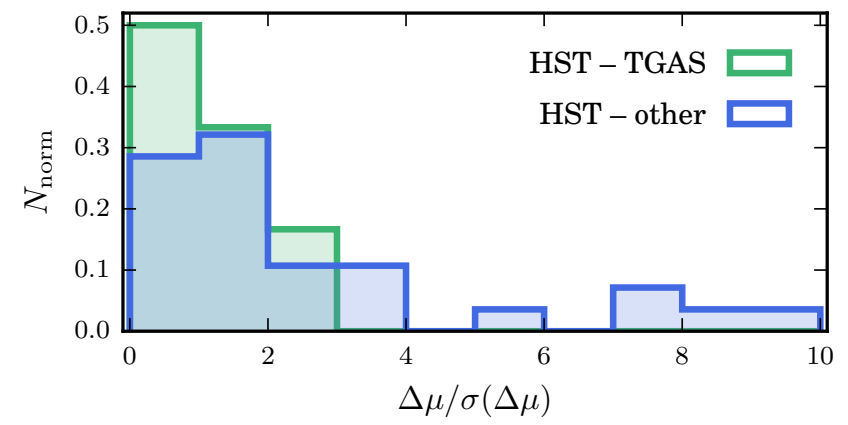

Figure 8. Normalised histograms of PM differences $\Delta \mu$ as a fraction of the uncertainty in the difference $\sigma(\Delta \mu)$ for all clusters, with RA PMs and Dec PMs considered independently. The blue histogram shows the offsets between HST PM measurements and other non-HST PM measurements, all taken from the literature (see Section 3). In some cases, the HST and nonHST measurements are discrepant at more than $5 \sigma$. The green histogram shows the offsets between literature HST PM measurements and our TGAS PM measurements. All HST and TGAS measurements agree within $3 \sigma$.

peated passages through the disk. This is borne out by observations: Ness et al. (2013) found a population of stars in their ARGOS study of the bulge that had properties consistent with NGC 6656, which could be extratidal stars from the cluster. Kunder et al. (2014) also found a candidate extratidal star of NGC 6656 in RAVE data.

\section{DISCUSSION AND CONCLUSIONS}

In this pilot study, we have identified a total of 20 members of 5 Galactic GCs in the TGAS catalogue from Gaia DR1 - 5 stars in NGC 104 (47 Tuc), 1 star in NGC 5272 (M 3), 5 stars in NGC 6121 (M4), 7 stars in NGC 6397, and 2 stars in NGC 6656 - and used these stars to determine the PMs and parallaxes of these clusters. These first Gaia PMs for GCs provide new insights into their dynamics. The parallaxes instead, while consistent with existing knowledge, are not yet competitive with other methods for determining GC distances.

We reviewed all existing PM measurements for the GCs in our final sample. Existing PM estimates for the same cluster do not generally agree with each other within the random errors; in some cases, measurements disagree by $5 \sigma$ or more (see Figure 8, blue histogram). Hence, the accuracy of the existing PM measurements is generally dominated by systematic errors, and not random errors, as is often the case in astrometry. By contrast, the TGAS catalogue combines measurements from Hipparcos and Gaia: two space missions with stable and well-calibrated platforms designed specifically to do astrometry. The main value of our TGAS analysis is therefore that it provides a homogeneous study of multiple GCs based on an astrometric catalogue with well-controlled systematics.

We have not explicitly included possible spatial correlations in TGAS PM errors (Gaia Collaboration et al. 2016; Lindegren et al. 2016) in our analysis. The effect of such correlations would be to underestimate the random error in the weighted average PM of a stellar sample (Kroupa \& Bastian 1997). Gaia DR1 has a nominal systematic positional accuracy of $\sim 0.3$ mas. Over the $\sim 25 \mathrm{yr}$ time baseline with the Hipparcos mission, this introduces a PM error of only $\sim 0.01$ mas/yr. van der Marel \& Sahlmann (2016) found the agreement between TGAS and HST PMs for the Magellanic Clouds to be significantly better than $\lesssim 0.1$ mas/yr. This further bounds the size of any possible PM systematics. Systematic PM errors in the TGAS PMs are therefore well below the size of the random PM errors for our GCs in Table 2 (which range from 0.29 to $1.49 \mathrm{mas} / \mathrm{yr}$ ).

The random PM errors from our analysis are comparable to those from many existing studies, but do not yet improve upon them. In general, the PM diagrams presented here show that our measurements are consistent with the "scatter clouds" of measurements from previous studies. A detailed assessment shows that our measurements are generally fully consistent with existing HST-based measurements (see Figure 8, green histogram), but not with all existing ground-based measurements. This agrees with the findings of van der Marel \& Sahlmann (2016), who found excellent agreement between measurements of the PMs of the Magellanic Clouds from TGAS and HST. We therefore conclude that: (1) since TGAS and HST use very different methods for astrometry, our GC results provide further external validation of both data sets and their underlying approaches; and (2) many ground-based GC PM measurements suffer from systematic uncertainties in excess of the random errors.

We also combined our PMs with literature RVs and distances to calculate absolute Galactocentric space motions for the clusters, corrected for peculiar motion of the Sun relative to the LSR and for rotation of the LSR about the Galactic Centre. Our space motions are broadly consistent with previous orbital analyses.

Our results highlight the promise of future Gaia data releases for the determination of parallaxes and PMs in Galactic GCs. Comparing parallax distances from Gaia with previous photometric and dynamical distance estimates will pro- 
vide crucial verification of all three methods, and improved distances and PMs will further constrain GC orbits. With future releases, Gaia PMs will be able to resolve the internal motions of nearby Galactic GCs as well as measure global motions as we have done here.

Gaia PMs and HST PMs are highly complementary, as the two observatories have different strengths. At present, the majority of HST PMs for the inner GCs are relative, due to the difficulty of finding sufficient fixed background sources in the crowded GC fields that can be used to create an absolute reference frame (Bellini et al. 2014). This does not affect velocity dispersion or anisotropy determination (Watkins et al. 2015 b) but does not allow for measurement of rotation, or indeed the global motion of the GC. Gaia PMs are absolute, so they will provide crucial constraints on cluster rotation and on their global motions. By contrast, HST can measure PMs down to fainter magnitudes, so it can go deeper for the nearby clusters, and can measure PMs for more distant clusters than Gaia will be able to achieve. Thus, HST PMs will be crucial for constraining the shape of the outer MW halo. HST will also provide more accurate astrometry in the crowded centres of clusters (though these regions are challenging even for $H S T)$.

For now, HST remains the best source of GC PMs, however, in the future the the best choice will depend on the particular science goals. Combined together, Gaia and HST PMs will provide the greatest coverage, depth, and accuracy of all and will revolutionise our understanding of both individual clusters and the entire MW GC population.

\section{ACKNOWLEDGEMENTS}

We thank the anonymous referee for a useful report. L.L.W. wishes to thank Erik Tollerud for useful discussions. This research has made use of Astropy ${ }^{10}$, a community-developed core Python package for Astronomy (Astropy Collaboration et al. 2013). This research has made use of NASA's Astrophysics Data System. This work has made use of data from the European Space Agency (ESA) space mission Gaia ${ }^{11}$, processed by the Gaia Data Processing and Analysis Consortium (DPAC) ${ }^{12}$. Funding for the DPAC has been provided by national institutions, in particular the institutions participating in the Gaia Multilateral Agreement.

\section{REFERENCES}

Anderson, J., \& King, I. R. 2003, AJ, 126, 772

Astraatmadja, T. L., \& Bailer-Jones, C. A. L. 2016, ApJ, 832, 137

Astropy Collaboration, Robitaille, T. P., Tollerud, E. J., et al. 2013, A\&A, 558, A33

Bedin, L. R., Piotto, G., King, I. R., \& Anderson, J. 2003, AJ, 126, 247

Bellini, A., Anderson, J., van der Marel, R. P., et al. 2014, ApJ, 797, 115

Bono, G., Stetson, P. B., Sanna, N., et al. 2008, ApJ, 686, L87

Casetti-Dinescu, D. I., Girard, T. M., Herrera, D., et al. 2007, AJ, 134, 195

Casetti-Dinescu, D. I., Girard, T. M., Jílková, L., et al. 2013, AJ, 146, 33

Casetti-Dinescu, D. I., Girard, T. M., Korchagin, V. I., van Altena, W. F., \& López, C. E. 2010, AJ, 140, 1282

Chaboyer, B. 1995, ApJ, 444, L9

Chen, D., Chen, L., \& Wang, J.-J. 2004, Chinese Physics Letters, 21, 1673

Cioni, M.-R. L., Bekki, K., Girardi, L., et al. 2016, A\&A, 586, A77

Cudworth, K. M. 1979, AJ, 84, 1312

-. 1986, AJ, 92, 348

Cudworth, K. M., \& Hanson, R. B. 1993, AJ, 105, 168
Cudworth, K. M., \& Rees, R. 1990, AJ, 99, 1491

Dinescu, D. I., Girard, T. M., \& van Altena, W. F. 1999a, AJ, 117, 1792

Dinescu, D. I., Girard, T. M., van Altena, W. F., \& López, C. E. 2003, AJ, 125,1373

Dinescu, D. I., Girard, T. M., van Altena, W. F., Mendez, R. A., \& Lopez, C. E. 1997, AJ, 114, 1014

Dinescu, D. I., van Altena, W. F., Girard, T. M., \& López, C. E. 1999b, AJ, 117,277

Dotter, A., Chaboyer, B., Jevremović, D., et al. 2008, ApJS, 178, 89

Ferraro, F. R., Messineo, M., Fusi Pecci, F., et al. 1999, AJ, 118, 1738

Freire, P. C., Camilo, F., Kramer, M., et al. 2003, MNRAS, 340, 1359

Gaia Collaboration, Brown, A. G. A., Vallenari, A., et al. 2016, A\&A, 595, A2

Geffert, M. 1998, A\&A, 340, 305

Gratton, R. G., Bragaglia, A., Carretta, E., et al. 2003, A\&A, 408, 529

Hansen, B. M. S., Anderson, J., Brewer, J., et al. 2007, ApJ, 671, 380

Harris, W. E. 1996, AJ, 112, 1487

Hendricks, B., Stetson, P. B., VandenBerg, D. A., \& Dall'Ora, M. 2012, AJ, 144,25

Heyl, J. S., Richer, H., Anderson, J., et al. 2012, ApJ, 761, 51

Høg, E., Fabricius, C., Makarov, V. V., et al. 2000, A\&A, 355, L27

Kalirai, J. S., Richer, H. B., Hansen, B. M., et al. 2004, ApJ, 601, 277

Kalirai, J. S., Anderson, J., Richer, H. B., et al. 2007, ApJ, 657, L93

Kallivayalil, N., van der Marel, R. P., \& Alcock, C. 2006, ApJ, 652, 1213

Kaluzny, J., Thompson, I. B., Rozyczka, M., et al. 2013, AJ, 145, 43

Krauss, L. M., \& Chaboyer, B. 2003, Science, 299, 65

Kroupa, P., \& Bastian, U. 1997, New A, 2, 77

Kunder, A., Stetson, P. B., Cassisi, S., et al. 2013, AJ, 146, 119

Kunder, A., Bono, G., Piffl, T., et al. 2014, A\&A, 572, A30

Kunder, A., Kordopatis, G., Steinmetz, M., et al. 2017, AJ, 153, 75

Küpper, A. H. W., Balbinot, E., Bonaca, A., et al. 2015, ApJ, 803, 80

Lee, J.-W., Kang, Y.-W., Lee, J., \& Lee, Y.-W. 2009, Nature, 462, 480

Lindegren, L., Lammers, U., Bastian, U., et al. 2016, A\&A, 595, A4

Mackey, A. D., \& Gilmore, G. F. 2004, MNRAS, 355, 504

Marconi, M., Caputo, F., Di Criscienzo, M., \& Castellani, M. 2003, ApJ, 596,299

Marino, A. F., Sneden, C., Kraft, R. P., et al. 2011, A\&A, 532, A8

Massari, D., Posti, L., Helmi, A., Fiorentino, G., \& Tolstoy, E. 2017, A\&A 598, L9

McLaughlin, D. E., \& van der Marel, R. P. 2005, ApJS, 161, 304

McMillan, P. J. 2011, MNRAS, 414, 2446

Michalik, D., Lindegren, L., \& Hobbs, D. 2015, A\&A, 574, A115

Milone, A. P., Villanova, S., Bedin, L. R., et al. 2006, A\&A, 456, 517

Monaco, L., Pancino, E., Ferraro, F. R., \& Bellazzini, M. 2004, MNRAS, 349,1278

Neeley, J. R., Marengo, M., Bono, G., et al. 2015, ApJ, 808, 11

Ness, M., Freeman, K., Athanassoula, E., et al. 2013, MNRAS, 432, 2092

Norris, J., \& Freeman, K. C. 1983, ApJ, 266, 130

Odenkirchen, M., Brosche, P., Geffert, M., \& Tucholke, H.-J. 1997, New A, 2, 477

Paez, E., Martinez Roger, C., \& Straniero, O. 1990, A\&AS, 84, 481

Pearson, S., Küpper, A. H. W., Johnston, K. V., \& Price-Whelan, A. M.

2015, ApJ, 799, 28

Peterson, R. C., \& Cudworth, K. M. 1994, ApJ, 420, 612

Peterson, R. C., Rees, R. F., \& Cudworth, K. M. 1995, ApJ, 443, 124

Reid, I. N., \& Gizis, J. E. 1998, AJ, 116, 2929

Robin, A. C., Reylé, C., Derrière, S., \& Picaud, S. 2003, A\&A, 409, 523

Salaris, M., Held, E. V., Ortolani, S., Gullieuszik, M., \& Momany, Y. 2007, A\&A, 476, 243

Sandage, A., \& Cacciari, C. 1990, ApJ, 350, 645

Scholz, R. D., Odenkirchen, M., \& Irwin, M. J. 1993, MNRAS, 264, 579

Schönrich, R., Binney, J., \& Dehnen, W. 2010, MNRAS, 403, 1829

Sirianni, M., Jee, M. J., Benítez, N., et al. 2005, PASP, 117, 1049

Thompson, I. B., Kaluzny, J., Rucinski, S. M., et al. 2010, AJ, 139, 329

van der Marel, R. P., \& Sahlmann, J. 2016, ApJ, 832, L23

Verde, L., Jimenez, R., \& Feeney, S. 2013, Physics of the Dark Universe, 2,

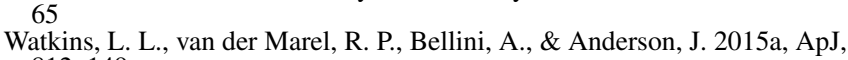
812,149

-. 2015b, ApJ, 803, 29

Webb, J. J., Leigh, N., Sills, A., Harris, W. E., \& Hurley, J. R. 2014, MNRAS, 442, 1569

Woodley, K. A., Goldsbury, R., Kalirai, J. S., et al. 2012, AJ, 143, 50

Wu, Z.-Y., Wang, J.-J., \& Chen, L. 2002, Chinese J. Astron. Astrophys., 2, 216

Zhu, L., Romanowsky, A. J., van de Ven, G., et al. 2016, MNRAS, 462, 4001

Zinn, R. 1985, ApJ, 293, 424

Zocchi, A., Gieles, M., Hénault-Brunet, V., \& Varri, A. L. 2016, MNRAS, 462,696

\footnotetext{
10 http://www.astropy.org

11 http://www.cosmos.esa.int/gaia
} 


\section{APPENDIX}

\section{ADDITIONAL FIGURES}

In Section 2.5, we determined whether stars identified as candidate cluster members are photometrically consistent with their nearest cluster. We showed the results of this analysis for NGC 6656 in Figure 1 as an example. Figure 9 shows the results for the other 14 clusters for which we performed this analysis.

In Section 2.6, we determined whether stars identified as candidate cluster members are consistent with predictions for the foreground population from the Besançon simulations. We showed the results of this analysis for NGC 6656 in Figure 2 as an example. Figure 10 and Figure 11 show the results for the other 10 clusters for which we performed this analysis.
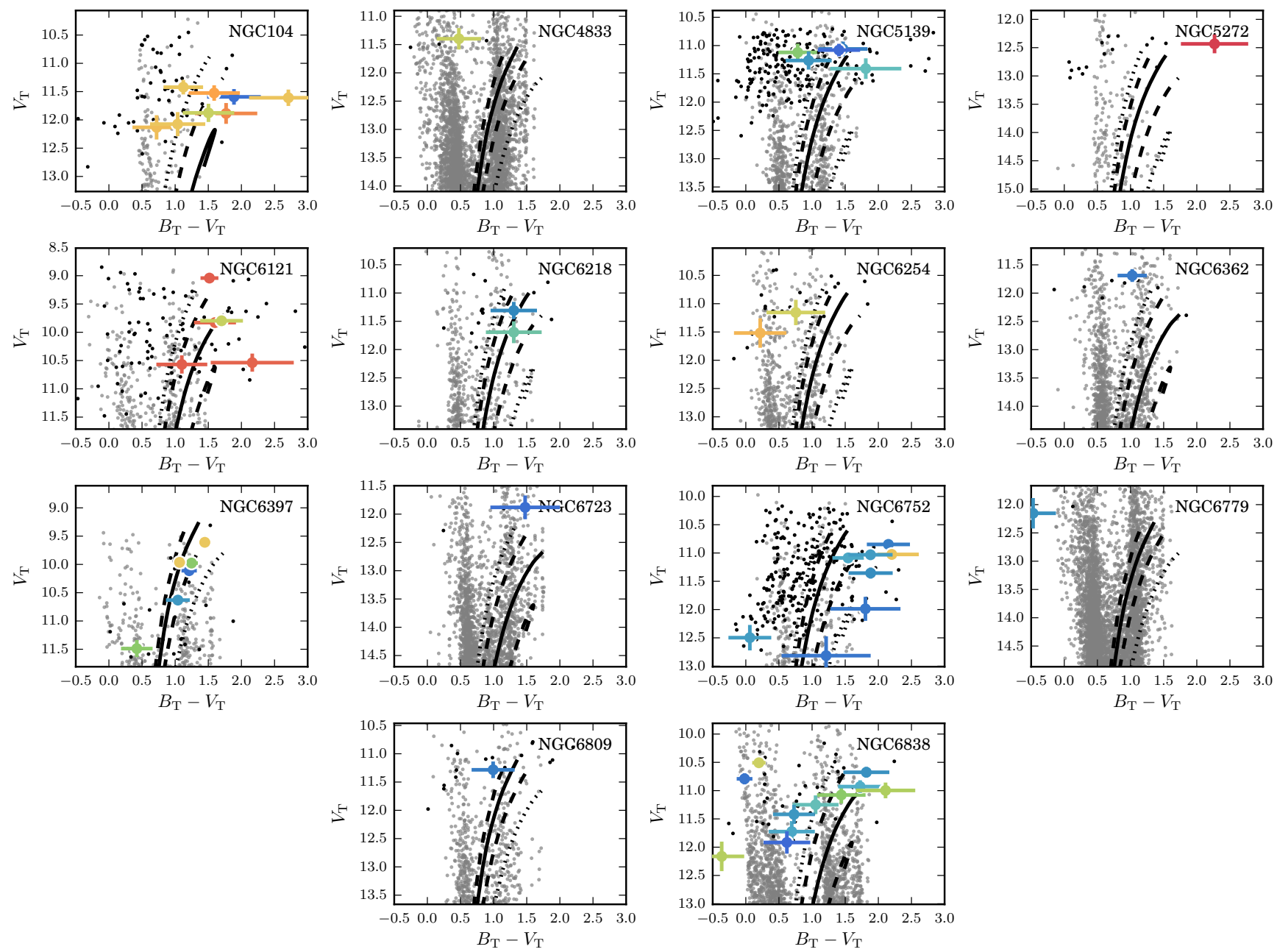

Figure 9. Same as Figure 1 for NGC 104, NGC 4833, NGC 5139, NGC 5272, NGC 6121, NGC 6218, NGC 6254, NGC 6362, NGC 6397, NGC 6723, NGC 6752, NGC 6779, NGC 6809, and NGC 6838. 

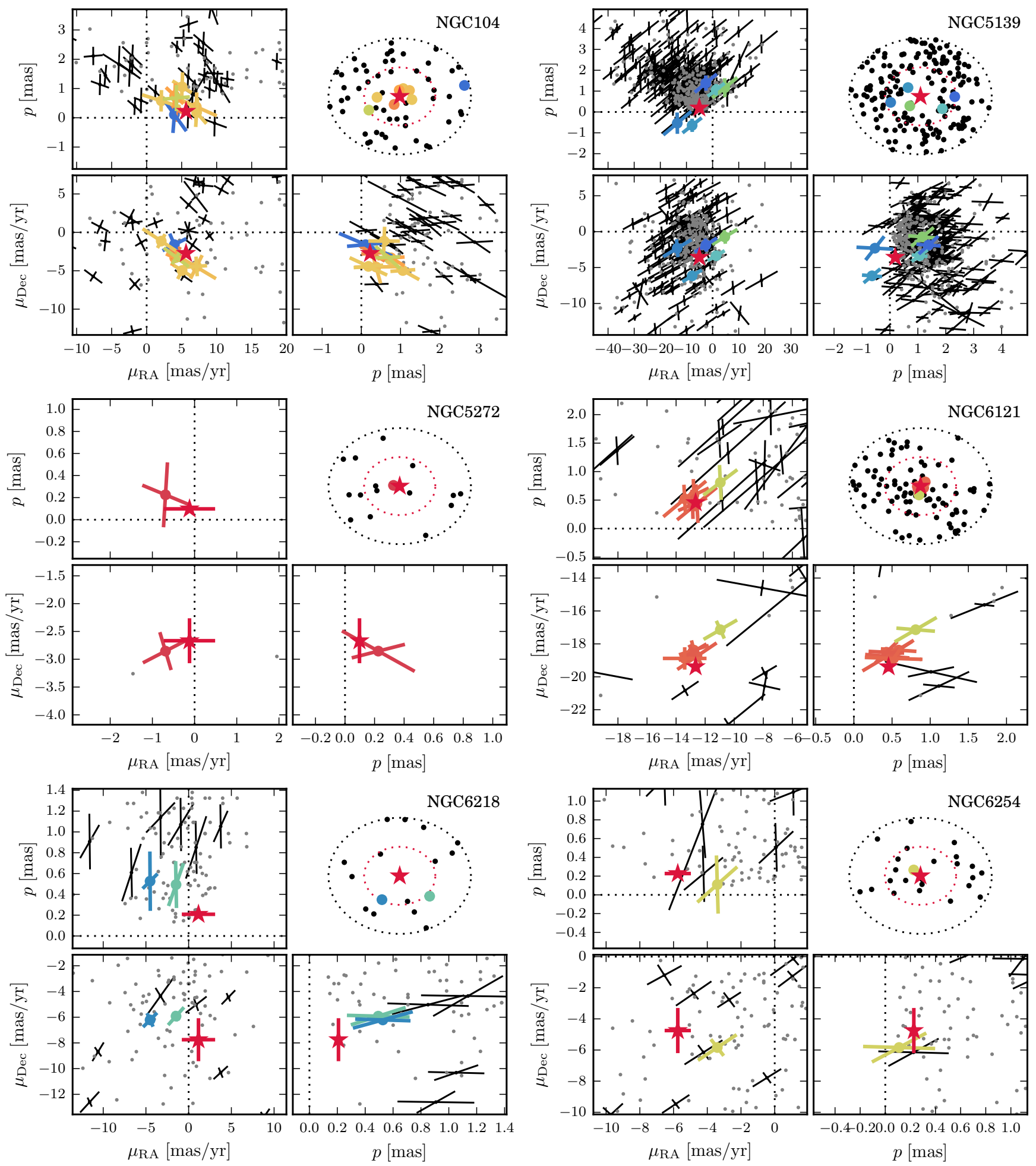

Figure 10. Same as Figure 2 for NGC 104, NGC 5139, NGC 5272, NGC 6121, NGC 6218, and NGC 6254. 

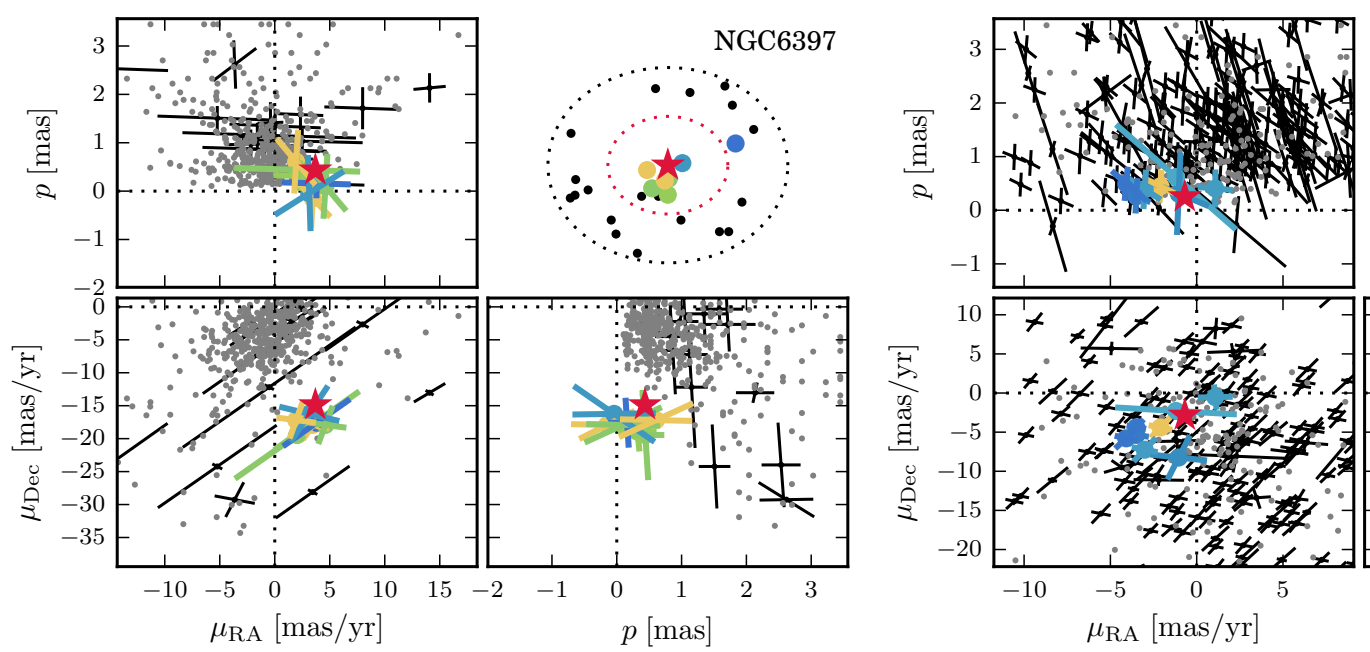

NGC6752
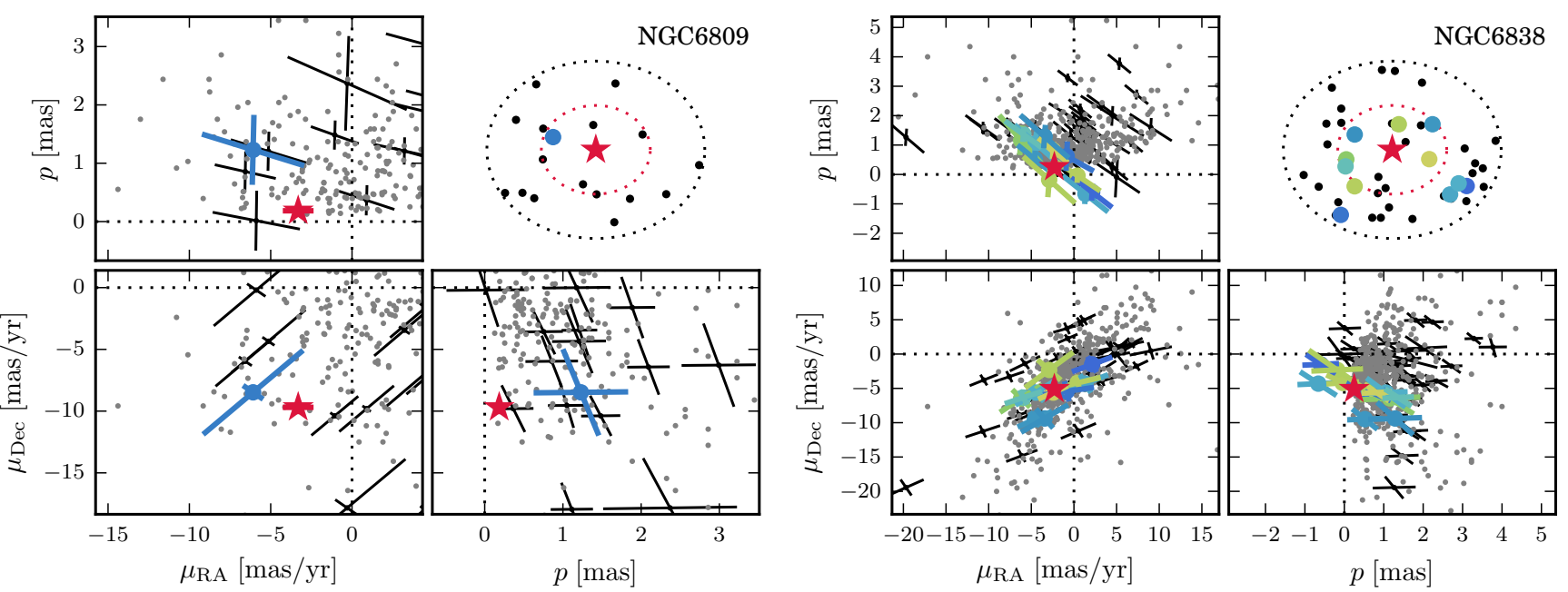

Figure 11. Same as Figure 2 for NGC 6397, NGC 6752, NGC 6809, and NGC 6838. 